\title{
On the faithfulness of parabolic cohomology as a Hecke module over a finite field
}

\author{
Gabor Wiese
}

19th November 2018

\begin{abstract}
This article exhibits conditions under which a certain parabolic group cohomology space over a finite field $\mathbb{F}$ is a faithful module for the Hecke algebra of cuspidal Katz modular forms over an algebraic closure of $\mathbb{F}$. These results can e.g. be applied to compute cuspidal Katz modular forms of weight one with methods of linear algebra over $\mathbb{F}$.

MSC Classif.: 11F25 (primary), 11F33, 11F67, 11 Y40 (secondary).
\end{abstract}

\section{Introduction}

The space $S_{k}\left(\Gamma_{1}(N), \mathbb{C}\right)$ of holomorphic modular cusp forms of weight $k \geq 2$ for the group $\Gamma_{1}(N)$ for some integer $N \geq 1$ is related to the parabolic group cohomology of $\Gamma_{1}(N)$ by the so-called Eichler-Shimura isomorphism (see e.g. [5], Theorem 12.2.2)

$$
S_{k}\left(\Gamma_{1}(N), \mathbb{C}\right) \oplus \overline{S_{k}\left(\Gamma_{1}(N), \mathbb{C}\right)} \cong H_{\mathrm{par}}^{1}\left(\Gamma_{1}(N), \mathbb{C}[X, Y]_{k-2}\right),
$$

where $\mathbb{C}[X, Y]_{k-2}$ denotes the $\mathbb{C}$-vector space of homogeneous polynomials of degree $k-2$ in two variables with the standard $\mathrm{SL}_{2}(\mathbb{Z})$-action. For the definition of the parabolic group cohomology see Section 2. On the modular forms, as well as on the group cohomology one disposes of natural Hecke operators, which are compatible with the Eichler-Shimura isomorphism. An immediate observation is that $H_{\text {par }}^{1}\left(\Gamma_{1}(N), \mathbb{C}[X, Y]_{k-2}\right)$ is a free module of rank 2 for the complex Hecke algebra $\mathbb{T}_{\mathbb{C}}$ generated by the Hecke operators in the endomorphism ring of $S_{k}\left(\Gamma_{1}(N), \mathbb{C}\right)$. This implies, in particular, that $H_{\text {par }}^{1}\left(\Gamma_{1}(N), \mathbb{C}[X, Y]_{k-2}\right)$ is a faithful $\mathbb{T}_{\mathbb{C} \text {-module. }}$

In general there is no analogue of the Eichler-Shimura isomorphism over finite fields. However, in the present article we show that the above observation generalises to cuspidal Katz modular forms over finite fields in certain cases.

Let now $\mathbb{T}_{\mathbb{F}_{p}}$ denote the $\mathbb{F}_{p}$-Hecke algebra generated by the Hecke operators inside the endomorphism ring of the cuspidal Katz modular forms $S_{k}\left(\Gamma_{1}(N), \mathbb{F}_{p}\right)$ of weight $k \geq 2$ for $\Gamma_{1}(N)$ over the field $\mathbb{F}_{p}$. In view of the observations from the Eichler-Shimura isomorphism two questions arise naturally. 
(a) Is $H_{\text {par }}^{1}\left(\Gamma_{1}(N), \mathbb{F}_{p}[X, Y]_{k-2}\right)$ a faithful $\mathbb{T}_{\mathbb{F}_{p}}$-module?

(b) Is $H_{\text {par }}^{1}\left(\Gamma_{1}(N), \mathbb{F}_{p}[X, Y]_{k-2}\right)$ free of rank 2 as a $\mathbb{T}_{\mathbb{F}_{p}}$-module?

A positive answer to Question (b) clearly implies a positive answer to (a).

Using $p$-adic Hodge theory (in particular the article [10), Edixhoven shows in Theorem 5.2 of [8] that Question (a) has a positive answer in the weight range $2 \leq k \leq p-1$. Emerton, Pollack and Weston were able to deduce from the fundamental work by Wiles on Fermat's last theorem that Question (b) is true locally at primes of the Hecke algebra which are $p$-ordinary and $p$-distinguished (see 9], Proposition 3.3.1) for $\mathbb{Z}_{p}$-coefficients and the full cohomology (i.e. not the parabolic subspace), from which the result follows for $\mathbb{F}_{p}$-coefficients and the full cohomology. We should mention that a positive answer to Question (a) with $\mathbb{Z}_{p}$-coefficients does not imply a positive answer over $\mathbb{F}_{p}$.

The main result of the present article is to answer Question (a) positively locally at $p$-ordinary primes of $\mathbb{T}_{\mathbb{F}_{p}}$ in the weight range $2 \leq k \leq p+1$ (see Corollary 6.9). A similar result for cuspidal modular forms for $\Gamma_{1}(N)$ with a Dirichlet character follows under certain rather weak assumptions (see Theorem (7.4). These results are obtained using techniques that were inspired by the special case $p=2$ of $[8$, Theorem 5.2.

Let us point out the following consequence, which presented the initial motivation for this study. If the answer to Question (a) is yes, we may compute $\mathbb{T}_{\mathbb{F}_{p}}$ via the Hecke operators on the finite dimensional $\mathbb{F}_{p}$-vector space $H_{\text {par }}^{1}\left(\Gamma_{1}(N), \mathbb{F}_{p}[X, Y]_{k-2}\right)$. This can be done explicitly on a computer and only requires linear algebra methods. The knowledge of the Hecke algebra is very interesting. One can, for example, try to verify whether it is a Gorenstein ring. This property is often implied when the Hecke algebra is isomorphic to a deformation ring (as e.g. in [24]).

Techniques from 8, Section 4, show how cuspidal Katz modular forms of weight one over $\overline{\mathbb{F}_{p}}$ can be related to weight $p$. We observe in Proposition 8.4 that the corresponding local factors of the Hecke algebra in weight $p$ are $p$ ordinary. Hence, a consequence of our result is that methods from $\mathbb{F}_{p}$-linear algebra can be used for the computation of weight one Katz cusp forms (see Theorem 8.5). The author carried out some such calculations and reported on them in 21.

It should be mentioned that if one is not interested in the Hecke algebra structure, but only in the systems of eigenvalues of Hecke eigenforms in $S_{k}\left(\Gamma_{1}(N), \overline{\mathbb{F}_{p}}\right)$, one can get them directly from $H^{1}\left(\Gamma_{1}(N), \mathbb{F}_{p}[X, Y]_{k-2}\right)$ for all $k \geq 2$ (see e.g. [22], Proposition 4.3.1). However, there may be more systems of eigenvalues in the group cohomology than in $S_{k}\left(\Gamma_{1}(N), \overline{\mathbb{F}_{p}}\right)$, but the extra ones can easily be identified.

This article is based on the use of group cohomology. The author studied in his thesis [22 and the article 23] relations to modular symbols and a certain cohomology group on the corresponding modular curve. All these agree for instance for any congruence subgroup of $\mathrm{SL}_{2}(\mathbb{Z})$ if the characteristic of the base field is $p \geq 5$ or in any characteristic for the group $\Gamma_{1}(N)$ with $N \geq 5$. 
We add some remarks on Katz modular forms. As a reference one can use [6], 14] or [5]. For $R=\mathbb{C}$, the Katz cuspidal modular forms are precisely the holomorphic cusp forms. Under the assumptions $N \geq 5$ and $k \geq 2$ one has $S_{k}\left(\Gamma_{1}(N), \mathbb{Z}[1 / N]\right) \otimes R \cong S_{k}\left(\Gamma_{1}(N), R\right)$ for any ring $R$ in which $N$ is invertible. In particular, this means that any Katz cusp form over $\overline{\mathbb{F}_{p}}$ can be lifted to characteristic zero in the same weight and level. The previous statement does not hold for weight $k=1$ in general.

Katz cusp forms over $\overline{\mathbb{F}_{p}}$ for a prime $p$ play an important rôle in a modified version of Serre's conjecture on modular forms (see [7]), as their use, on the one hand, gets rid of some character lifting problems, and on the other hand, allows one to minimise the weights of the modular forms. In particular, odd, irreducible Galois representations $\operatorname{Gal}(\overline{\mathbb{Q}} \mid \mathbb{Q}) \rightarrow \mathrm{GL}_{2}\left(\overline{\mathbb{F}_{p}}\right)$ which are unramified at $p$ are conjectured to correspond to Katz eigenforms of weight one.

Finally, we should mention that William Stein has implemented $\mathbb{F}_{p}$-modular symbols and the Hecke operators on them in Magma and Python. One could say that this article is also about what they compute.

\section{Acknowledgements}

This article has grown out of Chapter III of my thesis. I would like to thank my thesis advisor Bas Edixhoven for very helpful suggestions, and Theo van den Bogaart for sharing his insights in algebraic geometry.

\section{$1.1 \quad$ Notation}

We denote by $\operatorname{Mat}_{2}(\mathbb{Z})_{\neq 0}$ the semi-group of integral $2 \times 2$-matrices with nonzero determinant. On it one has Shimura's main involution $\left(\begin{array}{ll}a & b \\ c & d\end{array}\right)^{\iota}=\left(\begin{array}{cc}d & -b \\ -c & a\end{array}\right)$. For a ring $R$ the notation $R[X, Y]_{n}$ stands for the homogeneous polynomials of degree $n$ in the variables $X, Y$. We let

$$
V_{n}(R):=\operatorname{Sym}^{n}\left(R^{2}\right) \cong R[X, Y]_{n}
$$

which we equip with the natural left $\operatorname{Mat}_{2}(\mathbb{Z})_{\neq 0}$-semi-group action. If $V$ is an $R$-module, let $V^{\vee}$ be the dual $R$-module $\operatorname{Hom}_{R}(V, R)$.

As usual, the upper half plane is denoted by $\mathbb{H}$. Moreover, we shall use the standard congruence subgroups $\Gamma(N), \Gamma_{1}(N)$ and $\Gamma_{0}(N)$ of $\mathrm{SL}_{2}(\mathbb{Z})$ for an integer $N \geq 1$, which are defined to consist of the matrices $\left(\begin{array}{ll}a & b \\ c & d\end{array}\right) \in \mathrm{SL}_{2}(\mathbb{Z})$ reducing modulo $N$ to $\left(\begin{array}{ll}1 & 0 \\ 0 & 1\end{array}\right),\left(\begin{array}{ll}1 & * \\ 0 & 1\end{array}\right)$ resp. $\left(\begin{array}{l}* \\ 0\end{array} *\right)$.

\section{Parabolic group cohomology}

In this section we recall the definition of parabolic group cohomology and present some properties to be used in the sequel. We shall use standard facts on group cohomology without special reference. The reader can for example consult [3]. 


\section{Cohomology of $\mathrm{PSL}_{2}(\mathbb{Z})$}

The group $\mathrm{PSL}_{2}(\mathbb{Z})$ is freely generated by the matrix classes of $\sigma:=\left(\begin{array}{cc}0 & -1 \\ 1 & 0\end{array}\right)$ and $\tau:=\left(\begin{array}{cc}1 & -1 \\ 1 & 0\end{array}\right)$. A consequence is the following special case of the MayerVietoris sequence (see e.g. [13], p. 221). For any ring $R$ and any left $R\left[\mathrm{PSL}_{2}(\mathbb{Z})\right]$ module $M$ the sequence

$$
\begin{aligned}
0 \rightarrow M^{\mathrm{PSL}_{2}(\mathbb{Z})} & \rightarrow M^{\langle\sigma\rangle} \oplus M^{\langle\tau\rangle} \rightarrow M \\
& \rightarrow H^{1}\left(\mathrm{PSL}_{2}(\mathbb{Z}), M\right) \rightarrow H^{1}(\langle\sigma\rangle, M) \oplus H^{1}(\langle\tau\rangle, M) \rightarrow 0
\end{aligned}
$$

is exact and for all $i \geq 2$ one has isomorphisms

$$
H^{i}\left(\mathrm{PSL}_{2}(\mathbb{Z}), M\right) \cong H^{i}(\langle\sigma\rangle, M) \oplus H^{i}(\langle\tau\rangle, M)
$$

Corollary 2.1 Let $R$ be a ring and $\Gamma \leq \mathrm{PSL}_{2}(\mathbb{Z})$ be a subgroup of finite index such that all the orders of all stabiliser groups $\Gamma_{x}$ for $x \in \mathbb{H}$ are invertible in $R$. Then for all $R[\Gamma]$-modules $V$ one has $H^{1}(\Gamma, V)=M /\left(M^{\langle\sigma\rangle}+M^{\langle\tau\rangle}\right)$ with $M=\operatorname{Coind}_{\Gamma}^{\mathrm{PSL}_{2}(\mathbb{Z})}(V)$ and $H^{i}(\Gamma, V)=0$ for all $i \geq 2$.

Proof. We first recall that any non-trivially stabilised point $x$ of $\mathbb{H}$ is conjugate by an element of $\operatorname{PSL}_{2}(\mathbb{Z})$ to either $i$ or $\zeta_{3}=e^{2 \pi i / 3}$, whence all nontrivial stabiliser groups are of the form $g\langle\sigma\rangle g^{-1} \cap \Gamma$ or $g\langle\tau\rangle g^{-1} \cap \Gamma$ for some $g \in \mathrm{PSL}_{2}(\mathbb{Z})$. From Mackey's formula (see e.g. [23]) one obtains

$$
H^{i}\left(\langle\sigma\rangle, \operatorname{Coind}_{\Gamma}^{\mathrm{PSL}_{2}(\mathbb{Z})} V\right) \cong \prod_{g \in \Gamma \backslash \mathrm{PSL}_{2}(\mathbb{Z}) /\langle\sigma\rangle} H^{i}\left(g\langle\sigma\rangle g^{-1} \cap \Gamma, V\right)
$$

for all $i$ and a similar result for $\tau$. Due to the invertibility assumption the result follows from Shapiro's lemma and Equations (2.2) and (2.3).

The assumptions of the proposition are for instance always satisfied if $R$ is a field of characteristic not 2 or 3 . They also hold for $\Gamma_{1}(N)$ with $N \geq 4$ over any ring.

\section{Definition of parabolic group cohomology}

Let $R$ be a ring, $\Gamma \leq \mathrm{PSL}_{2}(\mathbb{Z})$ a subgroup of finite index and $T=\tau \sigma=\left(\begin{array}{ll}1 & 1 \\ 0 & 1\end{array}\right)$. One defines the parabolic group cohomology group for the $R[\Gamma]$-module $V$ as the kernel of the restriction map in

$$
0 \rightarrow H_{\mathrm{par}}^{1}(\Gamma, V) \rightarrow H^{1}(\Gamma, V) \stackrel{\mathrm{res}}{\longrightarrow} \prod_{g \in \Gamma \backslash \mathrm{PSL}_{2}(\mathbb{Z}) /\langle T\rangle} H^{1}\left(\Gamma \cap\left\langle g T g^{-1}\right\rangle, V\right) .
$$

The definition of parabolic cohomology is compatible with Shapiro's lemma, i.e. Equation (2.4) is isomorphic to

$$
0 \rightarrow H_{\mathrm{par}}^{1}\left(\mathrm{PSL}_{2}(\mathbb{Z}), M\right) \rightarrow H^{1}\left(\mathrm{PSL}_{2}(\mathbb{Z}), M\right) \stackrel{\text { res }}{\longrightarrow} H^{1}(\langle T\rangle, M)
$$

with $M=\operatorname{Coind}_{\Gamma}^{\mathrm{PSL}_{2}(\mathbb{Z})} V=\operatorname{Hom}_{R[\Gamma]}\left(R\left[\operatorname{PSL}_{2}(\mathbb{Z})\right], V\right)$, as one sees using e.g. Mackey's formula as in the proof of Corollary 2.1 
Proposition 2.2 Let $R$ be a ring and $\Gamma \leq \mathrm{PSL}_{2}(\mathbb{Z})$ be a subgroup of finite index such that all the orders of all stabiliser groups $\Gamma_{x}$ for $x \in \mathbb{H}$ are invertible in $R$. Then for all $R[\Gamma]$-modules $V$ the sequence

$0 \rightarrow H_{\mathrm{par}}^{1}(\Gamma, V) \rightarrow H^{1}(\Gamma, V) \stackrel{\mathrm{res}}{\longrightarrow} \prod_{g \in \Gamma \backslash \mathrm{PSL}_{2}(\mathbb{Z}) /\langle T\rangle} H^{1}\left(\Gamma \cap\left\langle g T g^{-1}\right\rangle, V\right) \rightarrow V_{\Gamma} \rightarrow 0$

is exact.

Proof. Due to the assumptions we may apply Corollary 2.1 The restriction map in Equation (2.5) thus becomes

$$
M /\left(M^{\langle\sigma\rangle}+M^{\langle\tau\rangle}\right) \stackrel{m \mapsto(1-\sigma) m}{\longrightarrow} M /(1-T) M,
$$

since $H^{1}(\langle T\rangle, M) \cong M /(1-T) M$. The isomorphism $M \cong\left(R\left[\mathrm{PSL}_{2}(\mathbb{Z})\right] \otimes_{R} V\right)_{\Gamma}$ allows one to compute that the cokernel of this map is $V_{\Gamma}$, the $\Gamma$-coinvariants.

\section{The module $V_{n}(R)$}

Let $R$ be a ring. Recall from Notation 1.1 that we put $V_{n}(R)=\operatorname{Sym}^{n}\left(R^{2}\right) \cong$ $R[X, Y]_{n}$.

Proposition 2.3 Suppose that $n$ ! is invertible in $R$. Then there is a perfect pairing

$$
V_{n}(R) \times V_{n}(R) \rightarrow R
$$

of R-modules, which induces an isomorphism $V_{n}(R) \rightarrow V_{n}(R)^{\vee}$ of R-modules respecting the $\operatorname{Mat}_{2}(\mathbb{Z})_{\neq 0}$-action which is given on $V_{n}(R)^{\vee}$ by $(M . \phi)(w)=$ $\phi\left(M^{\iota} w\right)$ for $M \in \operatorname{Mat}_{2}(\mathbb{Z})_{\neq 0}, \phi \in V_{n}(R)^{\vee}$ and $w \in V_{n}(R)$.

Proof. One defines the perfect pairing on $V_{n}(R)$ by first constructing a perfect pairing on $R^{2}$, which we consider as column vectors. One sets

$$
R^{2} \times R^{2} \rightarrow R,\langle v, w\rangle:=\operatorname{det}(v \mid w)=v_{1} w_{2}-v_{2} w_{1} .
$$

If $M$ is a matrix in $\operatorname{Mat}_{2}(\mathbb{Z})_{\neq 0}$, one checks easily that $\langle M v, w\rangle=\left\langle v, M^{\iota} w\right\rangle$. This pairing extends naturally to a pairing on the $n$-th tensor power of $R^{2}$. Due to the assumption on the invertibility of $n$ !, we may view $\operatorname{Sym}^{n}\left(R^{2}\right)$ as a submodule in the $n$-th tensor power, and hence obtain the desired pairing and the isomorphism of the statement.

Lemma 2.4 Let $n \geq 1$ be an integer, $t=\left(\begin{array}{cc}1 & N \\ 0 & 1\end{array}\right)$ and $t^{\prime}=\left(\begin{array}{ll}1 & 0 \\ N & 1\end{array}\right)$. If $n ! N$ is not a zero divisor in $R$, then for the t-invariants we have $V_{n}(R)^{\langle t\rangle}=\left\langle X^{n}\right\rangle$ and for the $t^{\prime}$-invariants $V_{n}(R)^{\left\langle t^{\prime}\right\rangle}=\left\langle Y^{n}\right\rangle$. If $n ! N$ is invertible in $R$, then the coinvariants are given by $V_{n}(R)_{\langle t\rangle}=V_{n}(R) /\left\langle Y^{n}, X Y^{n-1}, \ldots, X^{n-1} Y\right\rangle$ respectively $V_{n}(R)_{\left\langle t^{\prime}\right\rangle}=V_{n}(R) /\left\langle X^{n}, X^{n-1} Y, \ldots, X Y^{n-1}\right\rangle$. 
Proof. The action of $t$ is $t .\left(X^{n-i} Y^{i}\right)=X^{n-i}(N X+Y)^{i}$ and consequently $(t-1) \cdot\left(X^{n-i} Y^{i}\right)=\sum_{j=0}^{i-1} r_{i, j} X^{n-j} Y^{j}$ with $r_{i, j}=N^{i-j}\left(\begin{array}{c}i \\ j\end{array}\right)$, which is not a zero divisor, respectively invertible, by assumption. For $x=\sum_{i=0}^{n} a_{i} X^{n-i} Y^{i}$ we have $(t-1) \cdot x=\sum_{j=0}^{n-1} X^{n-j} Y^{j}\left(\sum_{i=j+1}^{n} a_{i} r_{i, j}\right)$. If $(t-1) \cdot x=0$, we conclude for $j=n-1$ that $a_{n}=0$. Next, for $j=n-2$ it follows that $a_{n-1}=0$, and so on, until $a_{1}=0$. This proves the statement on the $t$-invariants. The one on the $t^{\prime}$-invariants follows from symmetry. The claims on the coinvariants are proved in a very similar and straightforward way.

Proposition 2.5 Let $n \geq 1$ be an integer.

(a) If $n ! N$ is not a zero divisor in $R$, then the $R$-module of $\Gamma(N)$-invariants $V_{n}(R)^{\Gamma(N)}$ is zero.

(b) If $n ! N$ is invertible in $R$, then the $R$-module of $\Gamma(N)$-coinvariants $V_{n}(R)_{\Gamma(N)}$ is zero.

(c) Suppose that $\Gamma$ is a subgroup of $\mathrm{SL}_{2}(\mathbb{Z})$ such that reduction modulo p defines a surjection $\Gamma \rightarrow \mathrm{SL}_{2}\left(\mathbb{F}_{p}\right)$ (e.g. $\Gamma(N), \Gamma_{1}(N), \Gamma_{0}(N)$ for $\left.p \nmid N\right)$. Suppose moreover that $1 \leq n \leq p$ if $p>2$, and $n=1$ if $p=2$. Then one has $V_{n}\left(\mathbb{F}_{p}\right)^{\Gamma}=0=V_{n}\left(\mathbb{F}_{p}\right)_{\Gamma}$.

Proof. As $\Gamma(N)$ contains the matrices $t$ and $t^{\prime}$, Lemma 2.4 already finishes Parts (a) and (b). The only part of (c) that is not yet covered is when the degree is $n=p>2$. Since $V_{p}\left(\mathbb{F}_{p}\right)$ is naturally isomorphic to $U_{1}$, Proposition 4.1 gives the exact sequence of $\Gamma$-modules $0 \rightarrow V_{1}\left(\mathbb{F}_{p}\right) \rightarrow V_{p}\left(\mathbb{F}_{p}\right) \rightarrow V_{p-2}\left(\mathbb{F}_{p}\right) \rightarrow 0$. It suffices to take invariants respectively coinvariants to obtain the result.

\section{Torsion-freeness and base change properties}

Herremans has computed a torsion-freeness result like the following proposition in [12, Proposition 9. Here we give a short and conceptual proof of a slightly more general statement. The way of approach was suggested by Bas Edixhoven.

Proposition 2.6 Assume that $R$ is an integral domain of characteristic 0 such that $R / p R \cong \mathbb{F}_{p}$ for a prime $p$. Let $N \geq 1$ and $k \geq 2$ be integers and let $\Gamma \leq \mathrm{SL}_{2}(\mathbb{Z})$ be a subgroup containing $\Gamma(N)$ but not -1 such that the orders of the stabiliser subgroups $\Gamma_{x}$ for $x \in \mathbb{H}$ have order coprime to $p$. Then the following statements hold:

(a) $H^{1}\left(\Gamma, V_{k-2}(R)\right) \otimes_{R} \mathbb{F}_{p} \cong H^{1}\left(\Gamma, V_{k-2}\left(\mathbb{F}_{p}\right)\right)$.

(b) If $k=2$, then $H^{1}\left(\Gamma, V_{k-2}(R)\right)[p]=0$. If $k \geq 3$, then $H^{1}\left(\Gamma, V_{k-2}(R)\right)[p]=$ $V_{k-2}\left(\mathbb{F}_{p}\right)^{\Gamma}$. In particular, if $p \nmid N$, then $H^{1}\left(\Gamma, V_{k-2}(R)\right)[p]=0$ for all $k \in\{2, \ldots, p+2\}$.

(c) If $k=2$, or if $k \in\{3, \ldots, p+2\}$ and $p \nmid N$, then $H_{\mathrm{par}}^{1}\left(\Gamma, V_{k-2}(R)\right) \otimes_{R} \mathbb{F}_{p} \cong$ $H_{\text {par }}^{1}\left(\Gamma, V_{k-2}\left(\mathbb{F}_{p}\right)\right)$. 
Proof. Let us first notice that the sequence

$$
0 \rightarrow V_{k-2}(R) \stackrel{\cdot p}{\rightarrow} V_{k-2}(R) \rightarrow V_{k-2}\left(\mathbb{F}_{p}\right) \rightarrow 0
$$

of $R[\Gamma]$-modules is exact. The associated long exact sequence gives rise to the short exact sequence

$$
0 \rightarrow H^{i}\left(\Gamma, V_{k-2}(R)\right) \otimes \mathbb{F}_{p} \rightarrow H^{i}\left(\Gamma, V_{k-2}\left(\mathbb{F}_{p}\right)\right) \rightarrow H^{i+1}\left(\Gamma, V_{k-2}(R)\right)[p] \rightarrow 0
$$

for every $i \geq 0$. Exploiting this sequence for $i=1$ immediately yields Part (a), since any $H^{2}$ of $\Gamma$ is zero by Corollary 2.1. Part (b) is a direct consequence of the case $i=0$ and Proposition 2.5.

We have the exact commutative diagram

$$
\begin{aligned}
& 0 \longrightarrow H^{1}\left(\Gamma, V_{k-2}(R)\right) \stackrel{\cdot p}{\longrightarrow} H^{1}\left(\Gamma, V_{k-2}(R)\right) \longrightarrow H^{1}\left(\Gamma, V_{k-2}\left(\mathbb{F}_{p}\right)\right) \longrightarrow 0 \\
& \downarrow \quad \downarrow \quad \downarrow \\
& 0 \rightarrow \prod_{g} H^{1}\left(D_{g}, V_{k-2}(R)\right) \stackrel{\cdot p}{\rightarrow} \prod_{g} H^{1}\left(D_{g}, V_{k-2}(R)\right) \rightarrow \prod_{g} H^{1}\left(D_{g}, V_{k-2}\left(\mathbb{F}_{p}\right)\right) \rightarrow 0 \\
& \begin{array}{cc}
\downarrow & \downarrow \\
\left(V_{k-2}(R)\right)_{\Gamma} & \cdot p \\
\downarrow & \left(V_{k-2}(R)\right)_{\Gamma} \\
0 & \downarrow \\
& 0
\end{array}
\end{aligned}
$$

where the products are taken over $g \in \Gamma \backslash \mathrm{PSL}_{2}(\mathbb{Z}) /\langle T\rangle$, and $D_{g}=\Gamma \cap\left\langle g T g^{-1}\right\rangle$. The exactness of the first row is the contents of Parts (a) and (b). That the columns are exact follows from Proposition 2.2. The zero on the right of the second row is due to the fact that $D_{g}$ is free on one generator. That generator is of the form $g\left(\begin{array}{ll}1 & r \\ 0 & 1\end{array}\right) g^{-1}$ with $r \mid N$, so that $r$ is invertible in $\mathbb{F}_{p}$. The zero on the left is trivial for $k=2$ and for $3 \leq k \leq p+2$ it is a consequence of Lemma 2.4. Part (c) now follows from the snake lemma and Proposition 2.5. which implies that the bottom map is an injection.

\section{Hecke action}

Hecke operators conceptually come from Hecke correspondences on modular curves respectively modular stacks. They are best described on the moduli interpretations (see e.g. [5], 3.2 and 7.3). All Hecke operators that we will encounter in this article arise like this. This section presents Hecke operators on group cohomology and the principal result is the behaviour of the Hecke operators with respect to Shapiro's lemma. That result was obtained by Ash and Stevens (1], Lemma 2.2). Here, however, we avoid their rather heavy language of weakly compatible Hecke pairs. Instead, the description of Hecke operators on group cohomology is used which comes directly from the Hecke correspondences (formally one has to work on the modular stacks with locally constant coefficients, in case of non-trivially stabilised points). For the description we follow [5], 12.4 . 


\section{Hecke operators on group cohomology}

Let $R$ be a ring, $\alpha \in \operatorname{Mat}_{2}(\mathbb{Z})_{\neq 0}$ and $\Gamma \leq \mathrm{PSL}_{2}(\mathbb{Z})$ be a subgroup containing some $\Gamma(N)$. We use the notations $\Gamma_{\alpha}:=\Gamma \cap \alpha^{-1} \Gamma \alpha$ and $\Gamma^{\alpha}:=\Gamma \cap \alpha \Gamma \alpha^{-1}$, where we consider $\alpha^{-1}$ as an element of $\mathrm{GL}_{2}(\mathbb{Q})$. Both groups are commensurable with $\Gamma$.

Suppose that $V$ is an $R$-module with a $\operatorname{Mat}_{2}(\mathbb{Z})_{\neq 0}$-semi-group action which restricts to an action by $\Gamma$. The Hecke operator $T_{\alpha}$ acting on group cohomology is the composite

$$
H^{1}(\Gamma, V) \stackrel{\text { res }}{\longrightarrow} H^{1}\left(\Gamma^{\alpha}, V\right) \stackrel{\text { conj }_{\alpha}}{\longrightarrow} H^{1}\left(\Gamma_{\alpha}, V\right) \stackrel{\text { cores }}{\longrightarrow} H^{1}(\Gamma, V) .
$$

The first map is the usual restriction, and the third one is the so-called corestriction, which one also finds in the literature under the name transfer. We explicitly describe the second map on non-homogeneous cocycles (cf. [5], p. 116):

$$
\operatorname{conj}_{\alpha}: H^{1}\left(\Gamma^{\alpha}, V\right) \rightarrow H^{1}\left(\Gamma_{\alpha}, V\right), \quad c \mapsto\left(g_{\alpha} \mapsto \alpha^{\iota} . c\left(\alpha g_{\alpha} \alpha^{-1}\right)\right) .
$$

There is a similar description on the parabolic subspace and the two are compatible. The following formula can also be found in [5], p. 116, and 20, Section 8.3.

Proposition 3.1 Suppose that $\Gamma \alpha \Gamma=\bigcup_{i=1}^{n} \Gamma \delta_{i}$ is a disjoint union. Then the Hecke operator $T_{\alpha}$ acts on $H^{1}(\Gamma, V)$ and $H_{\mathrm{par}}^{1}(\Gamma, V)$ by sending the nonhomogeneous cocyle $c$ to $T_{\alpha} c$ defined by

$$
\left(T_{\alpha} c\right)(g)=\sum_{i=1}^{n} \delta_{i}^{\iota} c\left(\delta_{i} g \delta_{j(i)}^{-1}\right)
$$

for $g \in \Gamma$. Here $j(i)$ is the index such that $\delta_{i} g \delta_{j(i)}^{-1} \in \Gamma$.

Proof. We only have to describe the corestriction explicitly. For that we notice that one has $\Gamma=\bigcup_{i=1}^{n} \Gamma_{\alpha} g_{i}$ with $\alpha g_{i}=\delta_{i}$. Furthermore the corestriction of a non-homogeneous cocycle $u \in H^{1}\left(\Gamma_{\alpha}, V\right)$ is the cocycle cores $(u)$ uniquely given by

$$
\operatorname{cores}(u)(g)=\sum_{i=1}^{n} g_{i}^{-1} u\left(g_{i} g g_{j(i)}^{-1}\right)
$$

for $g \in \Gamma$. Combining with the explicit description of the map $\operatorname{conj}_{\alpha}$ yields the result.

Suppose now that $\Gamma=\Gamma_{1}(N)$ (resp. $\left.\Gamma=\Gamma_{0}(N)\right)$. For a positive integer $n$, the Hecke operator $T_{n}$ is $\sum_{\alpha} T_{\alpha}$, where the sum runs through a system of representatives of the double cosets $\Gamma \backslash \Delta^{n} / \Gamma$ for the set $\Delta^{n}$ of matrices $\left(\begin{array}{ll}a & b \\ c & d\end{array}\right) \in \operatorname{Mat}_{2}(\mathbb{Z})_{\neq 0}$ of determinant $n$ such that $\left(\begin{array}{ll}a & b \\ c & d\end{array}\right) \equiv\left(\begin{array}{ll}1 & * \\ 0 & *\end{array}\right) \bmod N\left(\operatorname{resp} .\left(\begin{array}{ll}a & b \\ c & d\end{array}\right) \equiv\left(\begin{array}{ll}* & * \\ 0 & *\end{array}\right) \bmod N\right)$. For a prime $p$ one has $T_{p}=T_{\alpha}$ with $\alpha=\left(\begin{array}{ll}1 & 0 \\ 0 & p\end{array}\right)$. If $\Gamma=\Gamma_{1}(N)$ and the integer $d$ is coprime to $N$, the diamond operator $\langle d\rangle$ is $T_{\alpha}$ for any matrix $\alpha \in \mathrm{SL}_{2}(\mathbb{Z})$ whose reduction modulo $N$ is $\left(\begin{array}{cc}d^{-1} & 0 \\ 0 & d\end{array}\right)$. The diamond operator gives a group action by $(\mathbb{Z} / N \mathbb{Z})^{*}$ (with -1 acting trivially). If the level is $N M$ with $(N, M)=1$, then we can separate the diamond operator into two parts $\langle d\rangle=\langle d\rangle_{M} \times\langle d\rangle_{N}$, 
corresponding to $\mathbb{Z} / N M \mathbb{Z} \cong \mathbb{Z} / M \mathbb{Z} \times \mathbb{Z} / N \mathbb{Z}$. The Hecke and diamond operators satisfy the "usual" Euler product and one has the formulae $T_{n} T_{m}=T_{n m}$ for any pair of coprime integers $n, m$ and $T_{p^{r+1}}=T_{p^{r}} T_{p}-p^{k-1}\langle p\rangle T_{p^{r-1}}$ if $p \nmid N$, and $T_{p^{r+1}}=T_{p^{r}} T_{p}$ if $p \mid N$ for the action on $H^{1}\left(\Gamma_{1}(N), V_{k-2}\right)$.

\section{Hecke operators and Shapiro's lemma}

Let $N, M$ be coprime positive integers. In order to test compatibility of the Hecke operators with Shapiro's lemma we need to extend the $\Gamma_{1}(N)$-action on $\operatorname{Coind}_{\Gamma_{1}(N M)}^{\Gamma_{1}(N)}(V)$ to a $\operatorname{Mat}_{2}(\mathbb{Z})_{\neq 0}$-semi-group action "in the right way". For that we define the $R$-module $\mathcal{W}(M, V)$ as

$$
\left\{f \in \operatorname{Hom}_{R}\left(R\left[(\mathbb{Z} / M \mathbb{Z})^{2}\right], V\right) \mid f((u, v))=0 \forall(u, v) \text { s.t. }\langle u, v\rangle \neq \mathbb{Z} / M \mathbb{Z}\right\} .
$$

It carries the left $\operatorname{Mat}_{2}(\mathbb{Z})_{\neq 0}$-semi-group action $(g \cdot f)((u, v))=g f((u, v) g)$ for $f \in \mathcal{W}(M, V), g \in \operatorname{Mat}_{2}(\mathbb{Z})_{\neq 0}$ and $(u, v) \in(\mathbb{Z} / M \mathbb{Z})^{2}$.

Lemma 3.2 The homomorphism

$$
\mathcal{W}(M, V) \rightarrow \operatorname{Hom}_{R\left[\Gamma_{1}(N M)\right]}\left(R\left[\Gamma_{1}(N)\right], V\right), \quad f \mapsto(g \mapsto(g . f)((0,1)))
$$

is an isomorphism of left $\Gamma_{1}(N)$-modules (for the restricted action on $\mathcal{W}(M, V)$ ). In particular, $\mathcal{W}(M, V)$ is isomorphic to $\operatorname{Coind}_{\Gamma_{1}(N M)}^{\Gamma_{1}(N)}(V)$ as a left $\Gamma_{1}(N)$ module.

Proof. As $N$ and $M$ are coprime, reduction modulo $M$ defines a surjection from $\Gamma_{1}(N)$ onto $\mathrm{SL}_{2}(\mathbb{Z} / M \mathbb{Z})$. This implies that the map

$$
\Gamma_{1}(N M) \backslash \Gamma_{1}(N) \stackrel{A \mapsto(0,1) A \bmod M}{\longrightarrow}(\mathbb{Z} / M \mathbb{Z})^{2}
$$

is injective, and its image is the set of the $(u, v)$ with $\mathbb{Z} / M \mathbb{Z}=\langle u, v\rangle$. As the $\Gamma_{1}(N M)$-action on $V$ is the restriction of a $\Gamma_{1}(N)$-action, the coinduced module can be identified with $\operatorname{Hom}_{R}\left(R\left[\Gamma_{1}(N M) \backslash \Gamma_{1}(N)\right], V\right)$. From this the claimed isomorphism follows directly.

Lemma 3.3 Let $N, n$ be positive integers. We set

$$
\Delta_{1}(N)^{n}=\left\{\left(\begin{array}{ll}
a & b \\
c & d
\end{array}\right) \in \operatorname{Mat}_{2}(\mathbb{Z})_{\neq 0} \mid \operatorname{det}\left(\begin{array}{ll}
a & b \\
c & d
\end{array}\right)=n,\left(\begin{array}{ll}
a & b \\
c & d
\end{array}\right) \equiv\left(\begin{array}{cc}
1 & * \\
0 & *
\end{array}\right) \bmod N\right\} .
$$

There is the decomposition

$$
\Delta_{1}(N)^{n}=\bigcup_{a} \bigcup_{b} \Gamma_{1}(N) \sigma_{a}\left(\begin{array}{ll}
a & b \\
0 & d
\end{array}\right)
$$

where a runs through the integers such that $a>0,(a, N)=1, a d=n$ and $b$ through a system of representatives of $\mathbb{Z} / d \mathbb{Z}$. Here $\sigma_{a} \in \mathrm{SL}_{2}(\mathbb{Z})$ is a matrix reducing to $\left(\begin{array}{cc}a^{-1} & 0 \\ 0 & a\end{array}\right)$ modulo $N$. 
Proof. This is [20], Proposition 3.36.

The Shapiro map is the isomorphism on cohomology groups

$$
\text { Sh }: H^{1}\left(\Gamma_{1}(N), \mathcal{W}(M, V)\right) \rightarrow H^{1}\left(\Gamma_{1}(N M), V\right)
$$

which is induced by the homomorphism $\mathcal{W}(M, V) \rightarrow V$, sending $f$ to $f((0,1))$.

Proposition 3.4 For all integers $n, d \geq 1$ with $(d, N)=1$ we have

$$
T_{n} \circ \mathrm{Sh}=\mathrm{Sh} \circ T_{n} \quad \text { and } \quad\langle d\rangle_{N} \circ \mathrm{Sh}=\mathrm{Sh} \circ\langle d\rangle_{N} .
$$

Proof. We first prove the statement for $T_{n}$. For every integer $a>0$ dividing $n$ such that $(a, N)=1$ we choose a matrix $\sigma_{a}$ such that it reduces to $\left(\begin{array}{cc}a^{-1} & 0 \\ 0 & a\end{array}\right)$ modulo $N$. If $(a, M)=1$, then we also impose that $\sigma_{a}$ reduces to $\left(\begin{array}{cc}a^{-1} & 0 \\ 0 & a\end{array}\right)$ modulo $M$. If $(a, M) \neq 1$, then we want $\sigma_{a} \equiv\left(\begin{array}{ll}1 & 0 \\ 0 & 1\end{array}\right)$ modulo $M$. A simple calculation shows that $(0,1)\left(\sigma_{a}\left(\begin{array}{ll}a & b \\ 0 & d\end{array}\right)\right)^{\iota}$ is congruent to $(0,1)$ modulo $M$ if $(a, M)=1$ respectively to $(0, a)$ if $(a, M) \neq 1$.

A set of explicit coset representatives of $\Gamma_{1}(N M) \backslash \Delta_{1}(N M)^{n}$ is given by Lemma 3.3 as a subset of coset representatives of $\Gamma_{1}(N) \backslash \Delta_{1}(N)^{n}$, namely of those with $(a, M)=1$.

Let now $c \in H^{1}\left(\Gamma_{1}(N), \mathcal{W}(M, V)\right)$ be a cocycle. Then by Proposition 3.1 and the definition of the $\operatorname{Mat}_{2}(\mathbb{Z})_{\neq 0}$-action on $\mathcal{W}(M, V)$ we have for $g \in \Gamma_{1}(N M)$

$$
\left(\operatorname{Sh}\left(T_{n} c\right)\right)(g)=\sum_{\delta} \delta^{\iota}\left(c\left(\delta g \widetilde{\delta}^{-1}\right)\left((0,1) \delta^{\iota}\right)\right),
$$

where the sum runs over the above coset representatives for $\Gamma_{1}(N) \backslash \Delta_{1}(N)^{n}$ and $\widetilde{\delta}$ is chosen among these representatives such that $\delta g \widetilde{\delta}^{-1} \in \Gamma_{1}(N M)$. Moreover, we have

$$
\left(T_{n}(\operatorname{Sh}(c))\right)(g)=\sum_{\delta} \delta^{\iota}\left(c\left(\delta g \widetilde{\delta}^{-1}\right)((0,1))\right),
$$

where now the sum only runs through the subset described above. By what we have remarked right above $(0,1) \delta^{\iota}$ is congruent to $(0,1)$ modulo $M$ if and only if $(a, M)=1$. If $(a, M) \neq 1$, then $\langle a\rangle \neq \mathbb{Z} / M \mathbb{Z}$, but we have $(0,1) \delta^{\iota} \equiv$ $(0, a) \bmod N$. This proves the compatibility for $T_{n}$.

Since $\langle d\rangle_{N}$ only depends on $d$ modulo $N$, we may suppose that $d$ is congruent to 1 modulo $M$. We choose $\alpha=\sigma_{d}$ such that it reduces to $\left(\begin{array}{cc}d^{-1} & 0 \\ 0 & d\end{array}\right)$ modulo $N$ and to $\left(\begin{array}{ll}1 & 0 \\ 0 & 1\end{array}\right)$ modulo $M$. For $c \in H^{1}\left(\Gamma_{1}(N), \mathcal{W}(M, V)\right)$ and $g \in \Gamma_{1}(N M)$ the formula

$$
\left(\operatorname{Sh}\left(\langle d\rangle_{N} c\right)\right)(g)=\alpha^{\iota}\left(c\left(\alpha g \alpha^{-1}\right)\left((0,1) \alpha^{\iota}\right)\right)=\left(\langle d\rangle_{N}(\operatorname{Sh}(c))\right)(g)
$$

follows.

Proposition 3.5 For $(n, M)=1$ we define the $R\left[\operatorname{Mat}_{2}(\mathbb{Z})_{\neq 0}\right]$-isomorphism

$$
\operatorname{mult}_{n}: \mathcal{W}(M, V) \rightarrow \mathcal{W}(M, V), \quad f \mapsto((u, v) \mapsto f((n u, n v))) .
$$

Then we have

$$
\langle n\rangle_{M} \circ \mathrm{Sh}=\mathrm{Sh} \circ \operatorname{mult}_{n} .
$$


Proof. Let $\sigma \in \mathrm{SL}_{2}(\mathbb{Z})$ be a matrix reducing to $\left(\begin{array}{cc}n_{0}^{-1} & 0 \\ 0 & n\end{array}\right)$ modulo $M$ and to $\left(\begin{array}{ll}1 & 0 \\ 0 & 1\end{array}\right)$ modulo $N$. This means in particular that $\sigma \in \Gamma_{1}(N)$. Hence, for a cocycle $c \in H^{1}\left(\Gamma_{1}(N), \mathcal{W}(M, V)\right)$ we have

$$
\sigma^{-1} c\left(\sigma g \sigma^{-1}\right)=c(g)+(g-1) c\left(\sigma^{-1}\right)
$$

so that the equality $c\left(\sigma g \sigma^{-1}\right)=\sigma c(g)$ holds in $H^{1}\left(\Gamma_{1}(N), \mathcal{W}(M, V)\right)$.

We can now check the claim. First we have

$$
\left(\langle n\rangle_{M} \circ \mathrm{Sh}\right)(c)(g)=\sigma^{\iota}((\sigma . c(g))((0,1)))=c(g)((0,1) \sigma) .
$$

This agrees with $\left(\mathrm{Sh} \circ\right.$ mult $\left._{n}\right)(c)(g)=c(g)((0, n))$.

\section{Reduction to weight 2 for parabolic group co- homology}

This section deals with the reduction to weight 2 for parabolic group cohomology, by which we mean that the parabolic group cohomology over $\mathbb{F}_{p}$ for $\Gamma_{1}(N)$ with $p \nmid N$ and weight $3 \leq k \leq p+1$ is related to the weight two parabolic group cohomology of $\Gamma_{1}(N p)$. Hence, the parabolic group cohomology shows a behaviour similar to that of modular forms (cf. Proposition 5.1). Roughly speaking, the reduction to weight 2 on group cohomology comes from the decomposition of $\operatorname{Coind}_{\Gamma_{1}(N p)}^{\Gamma_{1}(N)}\left(\mathbb{F}_{p}\right)$ into simple $\mathbb{F}_{p}\left[\mathrm{SL}_{2}\left(\mathbb{F}_{p}\right)\right]$-modules. Those are precisely the $V_{d}\left(\mathbb{F}_{p}\right)$ for $0 \leq d \leq p-1$.

The contents of this section is already partly present in [1]. However, in that paper the parabolic subspace is not treated.

\section{Decomposition of $\mathcal{W}\left(p, \mathbb{F}_{p}\right)$ as $\mathbb{F}_{p}\left[\operatorname{Mat}_{2}(\mathbb{Z})_{\neq 0}\right]$-module}

We now relate the $\mathbb{F}_{p}\left[\operatorname{Mat}_{2}(\mathbb{Z})_{\neq 0}\right]$-modules $\mathcal{W}\left(p, \mathbb{F}_{p}\right)$ and $V_{d}\left(\mathbb{F}_{p}\right)$ for $0 \leq d \leq$ $p-1$. It is easy to check that evaluation of polynomials on $\mathbb{F}_{p}^{2}$ induces an isomorphism of $\mathbb{F}_{p}\left[\operatorname{Mat}_{2}(\mathbb{Z})_{\neq 0}\right]$-modules

$$
\mathbb{F}_{p}[X, Y] /\left(X^{p}-X, Y^{p}-Y\right) \cong \mathbb{F}_{p}^{\mathbb{F}_{p}^{2}}
$$

We can thus identify $\mathcal{W}\left(p, \mathbb{F}_{p}\right)$ with $\left\{f \in \mathbb{F}_{p}[X, Y] /\left(X^{p}-X, Y^{p}-Y\right) \mid f((0,0))=\right.$ $0\}$. Let $U_{d}\left(\mathbb{F}_{p}\right)$ be the subspace consisting of polynomial classes of degree $d$, i.e. those that satisfy $f(l x, l y)=l^{d} f(x, y)$ for all $l \in \mathbb{F}_{p}^{*}$. Note that the degree is naturally defined modulo $p-1$. It is clear that the natural $\operatorname{Mat}_{2}(\mathbb{Z})_{\neq 0}$-action respects the degree. We remark that the dimension of any $U_{d}$ is $p+1$. By collecting monomials we obtain

$$
\mathcal{W}\left(p, \mathbb{F}_{p}\right)=\bigoplus_{d=0}^{p-2} U_{d}\left(\mathbb{F}_{p}\right) .
$$

Furthermore, we dispose of the perfect bilinear pairing

$$
\mathcal{W}\left(p, \mathbb{F}_{p}\right) \times \mathcal{W}\left(p, \mathbb{F}_{p}\right) \rightarrow \mathbb{F}_{p}, \quad\langle f, g\rangle=\sum_{(a, b) \in \mathbb{F}_{p}^{2}} f(a, b) g(a, b) .
$$


It is elementary to check that $U_{d}\left(\mathbb{F}_{p}\right)$ pairs to zero with $U_{e}\left(\mathbb{F}_{p}\right)$ if $(p-1) \nmid(d+e)$. Hence, the restricted pairing $U_{d}\left(\mathbb{F}_{p}\right) \times U_{p-1-d}\left(\mathbb{F}_{p}\right) \rightarrow \mathbb{F}_{p}$ is perfect for $0 \leq d \leq$ $p-1$, as the dimensions of $U_{p-1-d}\left(\mathbb{F}_{p}\right)$ and $U_{d}\left(\mathbb{F}_{p}\right)$ are equal. Furthermore, $\mathbb{F}_{p}[X, Y]_{d}$ pairs to zero with $\mathbb{F}_{p}[X, Y]_{p-1-d}$. Consequently the induced pairing $U_{d}\left(\mathbb{F}_{p}\right) / V_{d}\left(\mathbb{F}_{p}\right) \times V_{p-1-d}\left(\mathbb{F}_{p}\right) \rightarrow \mathbb{F}_{p}$ is perfect. Composing with the map from Proposition 2.3. we obtain an isomorphism $U_{d}\left(\mathbb{F}_{p}\right) / V_{d}\left(\mathbb{F}_{p}\right) \rightarrow V_{p-1-d}\left(\mathbb{F}_{p}\right)$.

Proposition 4.1 Let $p$ be a prime and $d$ and integer with $0 \leq d \leq p-1$. The preceding construction gives the exact sequence

$$
0 \rightarrow V_{d}\left(\mathbb{F}_{p}\right) \rightarrow U_{d}\left(\mathbb{F}_{p}\right) \rightarrow V_{p-1-d}\left(\mathbb{F}_{p}\right) \rightarrow 0 .
$$

Moreover, the diagram

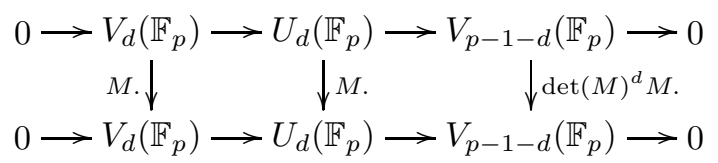

commutes for all $M \in \operatorname{Mat}_{2}(\mathbb{Z})_{\neq 0}$ whose reduction modulo $p$ is invertible. If $d>0$, then the diagram is also commutative for $M=\left(\begin{array}{ll}1 & 0 \\ 0 & p\end{array}\right)^{\iota}=\left(\begin{array}{ll}p & 0 \\ 0 & 1\end{array}\right)$, which means in particular that the right hand side vertical arrow is zero.

Proof. Only the commutativity need be checked. If $M$ is invertible modulo $p$, one immediately sees that the pairing on $\mathcal{W}\left(p, \mathbb{F}_{p}\right)$ is invariant under $M$, i.e. $\langle M f, M g\rangle=\langle f, g\rangle$ for all $f, g \in \mathcal{W}\left(p, \mathbb{F}_{p}\right)$. The commutativity is then clear

from Proposition 2.3 In order to treat $M=\left(\begin{array}{ll}p & 0 \\ 0 & 1\end{array}\right)$ one considers the basis of $U_{d}\left(\mathbb{F}_{p}\right)$ given by the monomials of degree $d$, which correspond to the embedding of $V_{d}\left(\mathbb{F}_{p}\right)$, together with the monomials $X^{i} Y^{p-1+d-i}$ for $d \leq i \leq p-1$. As the latter monomials all contain at least one factor of $X$ by assumption, they are killed by applying the matrix.

\section{Reduction to weight 2}

We introduce the following notation. Let $M$ be any $\mathbb{F}_{p}$-vector space on which the Hecke operators $T_{l}$ and the $p$-part of the diamond operators $\langle\cdot\rangle_{p}$ act. Suppose $d>0$. By $M[d]$ we mean $M$ with the action of the Hecke operator $T_{l}$ "twisted" to be $l^{d} T_{l}$ (in particular $T_{p}$ acts as zero). Furthermore, by $M(d)$ we denote the subspace on which $\langle l\rangle_{p}$ acts as $l^{d}$.

The non-parabolic part of the following proposition is also [1], Theorem 3.4.

Proposition 4.2 Let $p$ be a prime, $N \geq 4$ and $0<d \leq p-1$ integers such that $p \nmid N$. We have isomorphisms respecting the Hecke operators

$$
\begin{gathered}
H^{1}\left(\Gamma_{1}(N p), \mathbb{F}_{p}\right)(d) \cong H^{1}\left(\Gamma_{1}(N), U_{d}\left(\mathbb{F}_{p}\right)\right) \quad \text { and } \\
H_{\mathrm{par}}^{1}\left(\Gamma_{1}(N p), \mathbb{F}_{p}\right)(d) \cong H_{\mathrm{par}}^{1}\left(\Gamma_{1}(N), U_{d}\left(\mathbb{F}_{p}\right)\right) .
\end{gathered}
$$


Moreover, the sequences

$$
\begin{aligned}
0 \rightarrow H^{1}\left(\Gamma_{1}(N), V_{d}\left(\mathbb{F}_{p}\right)\right) \rightarrow H^{1}\left(\Gamma_{1}(N), U_{d}\left(\mathbb{F}_{p}\right)\right) \\
\\
\rightarrow H^{1}\left(\Gamma_{1}(N), V_{p-1-d}\left(\mathbb{F}_{p}\right)\right)[d] \rightarrow 0
\end{aligned}
$$

and

$$
\begin{aligned}
0 \rightarrow H_{\mathrm{par}}^{1}\left(\Gamma_{1}(N), V_{d}\left(\mathbb{F}_{p}\right)\right) \rightarrow H_{\mathrm{par}}^{1}\left(\Gamma_{1}(N), U_{d}\left(\mathbb{F}_{p}\right)\right) \\
\quad \rightarrow H_{\mathrm{par}}^{1}\left(\Gamma_{1}(N), V_{p-1-d}\left(\mathbb{F}_{p}\right)\right)[d] \rightarrow 0
\end{aligned}
$$

are exact and respect the Hecke operators.

Proof. The first statement follows from Propositions 3.4 and 3.5 together with the definition of $U_{d}\left(\mathbb{F}_{p}\right)$. The first exact sequence is part of the long exact sequence associated to the short exact sequence of Proposition 4.1 The commutative diagram in that proposition gives the twisting of the Hecke action in the exact sequences. This follows directly from the description of the Hecke operator on group cohomology.

For $d=p-1$ we have $U_{p-1}\left(\mathbb{F}_{p}\right)=V_{0}\left(\mathbb{F}_{p}\right) \oplus V_{p-1}\left(\mathbb{F}_{p}\right)$, from which the statements follow. So we now assume $d<p-1$, in particular $p \neq 2$. For the top sequence we only need to check that it is exact on the left and on the right. By Proposition 2.5 we have $H^{0}\left(\Gamma_{1}(N), V_{p-1-d}\left(\mathbb{F}_{p}\right)\right)=0$. The $H^{2}$-terms are trivial by Corollary 2.1 .

The exactness of the second sequence follows from the snake lemma, once we have established the exactness of

$$
\begin{aligned}
0 \rightarrow \prod_{c \text { cusps }} H^{1}\left(D_{c}, V_{d}\left(\mathbb{F}_{p}\right)\right) \rightarrow \prod_{c \text { cusps }} H^{1}\left(D_{c}, U_{d}\left(\mathbb{F}_{p}\right)\right) \\
\rightarrow \prod_{c \text { cusps }} H^{1}\left(D_{c}, V_{p-1-d}\left(\mathbb{F}_{p}\right)\right) \rightarrow 0,
\end{aligned}
$$

where $D_{c}$ is the stabiliser group of the cusp $c=g \infty$ with $g \in \mathrm{PSL}_{2}(\mathbb{Z})$. Hence, $D_{c}=g\langle T\rangle g^{-1} \cap \Gamma_{1}(N)$. This group is infinite cyclic generated by $g\left(\begin{array}{ll}1 & r \\ 0 & 1\end{array}\right) g^{-1}$ for some $r \in \mathbb{Z}$ dividing $N$ (see also the proof of Proposition 2.6). Hence, we have $H^{2}\left(D_{c}, V_{d}\left(\mathbb{F}_{p}\right)\right)=0$. We claim that the sequence

$$
\begin{aligned}
0 \rightarrow \prod_{c \text { cusps }} H^{0}\left(D_{c}, V_{d}\left(\mathbb{F}_{p}\right)\right) \rightarrow \prod_{c \text { cusps }} H^{0}\left(D_{c}, U_{d}\left(\mathbb{F}_{p}\right)\right) \\
\rightarrow \prod_{c \text { cusps }} H^{0}\left(D_{c}, V_{p-1-d}\left(\mathbb{F}_{p}\right)\right) \rightarrow 0
\end{aligned}
$$

is exact. Lemma 2.4 implies that $H^{0}\left(D_{c}, V_{d}\left(\mathbb{F}_{p}\right)\right)$ and $H^{0}\left(D_{c}, V_{p-1-d}\left(\mathbb{F}_{p}\right)\right)$ are one-dimensional. In order to finish the proof, it thus suffices to prove that $H^{0}\left(D_{c}, U_{d}\left(\mathbb{F}_{p}\right)\right)$ is (at least) of dimension 2. The elements $X^{d} \in U_{d}\left(\mathbb{F}_{p}\right)$ and $Y^{d}\left(1-X^{p-1}\right) \in U_{d}\left(\mathbb{F}_{p}\right)$ are invariant under $T$. Indeed,

$$
\begin{aligned}
& T . Y^{d}\left(1-X^{p-1}\right)=(X+Y)^{d}\left(1-X^{p-1}\right) \\
& =Y^{d}\left(1-X^{p-1}\right)+\sum_{i=1}^{d}\left(\begin{array}{l}
d \\
i
\end{array}\right) Y^{d-i} X^{i}\left(1-X^{p-1}\right)=Y^{d}\left(1-X^{p-1}\right),
\end{aligned}
$$


as in $U_{d}\left(\mathbb{F}_{p}\right)$ we have $X^{i}\left(1-X^{p-1}\right)=X^{i-1}\left(X-X^{p}\right)=0$ for $i>0$.

\section{$5 \quad$ Modular forms of weight 2 and level $N p$}

This section recalls the reduction to weight 2 for modular forms and establishes a link between parabolic group cohomology and modular forms via Jacobians of modular curves.

\section{Reduction to weight 2 for modular forms}

We recall some work of Serre as explained in [1], Sections 7 and 8, cf. also [7], Section 6 .

Let us now introduce notation that is used throughout the sequel of this article. We consider the modular curve $X_{1}(N p)$ over $\mathbb{Q}_{p}\left(\zeta_{p}\right)$ for a prime $p>2$ not dividing $N \geq 5$. It has a regular stable model $X$ over the ring $\mathbb{Z}_{p}\left[\zeta_{p}\right]$, see e.g. 16. Let $J$ denote the Néron model over $\mathbb{Z}_{p}\left[\zeta_{p}\right]$ of $J_{1}(N p)$, the Jacobian of $X_{1}(N p)$ over $\mathbb{Q}_{p}\left(\zeta_{p}\right)$. We let, following [11, Section 8,

$$
L=H^{0}\left(X, \Omega_{X / \mathbb{Z}_{p}\left[\zeta_{p}\right]}\right),
$$

where $\Omega_{X / \mathbb{Z}_{p}\left[\zeta_{p}\right]}$ is the dualising sheaf of $X$ of [4], Section I.2. By 11], Equation 8.2, we have for the special fibre $X_{\mathbb{F}_{p}}$ that

$$
\bar{L}:=H^{0}\left(X_{\mathbb{F}_{p}}, \Omega_{X_{\mathbb{F}_{p}} / \mathbb{F}_{p}}\right)=L \otimes_{\mathbb{Z}_{p}\left[\zeta_{p}\right]} \mathbb{F}_{p}
$$

On $L$ and $\bar{L}$ the $p$-part $\langle\cdot\rangle_{p}$ of the diamond operator acts. The principal result on $\bar{L}$ that we will need is the following (see Propositions 8.13 and 8.18 of [1]). The notation is as in Proposition 4.2.

Proposition 5.1 (Serre) Assume $3 \leq k \leq p, N \geq 5$ and $p \nmid N$. Then there is an isomorphism of $\mathbb{F}_{p}$-vector spaces

$$
\bar{L}(k-2) \cong S_{k}\left(\Gamma_{1}(N), \mathbb{F}_{p}\right) \oplus S_{p+3-k}\left(\Gamma_{1}(N), \mathbb{F}_{p}\right)[k-2]
$$

respecting the Hecke operators. Moreover, the sequence of Hecke modules

$$
0 \rightarrow S_{2}\left(\Gamma_{1}(N), \mathbb{F}_{p}\right)[p-1] \rightarrow \bar{L}(p-1) \rightarrow S_{p+1}\left(\Gamma_{1}(N), \mathbb{F}_{p}\right) \rightarrow 0
$$

is exact.

\section{Parabolic cohomology and the $p$-torsion of the Jacobian}

In order to compare Hecke algebras of cusp forms with those of parabolic group cohomology in characteristic $p$, we generalise the strategy of the second part of the proof of [8], Theorem 5.2. Hence, we wish to bring the Jacobian into the play, since it will enable us to pass from characteristic zero geometry to characteristic $p$. 
Lemma 5.2 There are isomorphisms

$$
\bar{L} \cong \operatorname{Cot}_{0}\left(J_{\mathbb{F}_{p}}^{0}\right) \cong \operatorname{Cot}_{0}\left(J_{\mathbb{F}_{p}}^{0}[p]\right)
$$

respecting the Hecke operators.

Proof. The first isomorphism is e.g. [7, Equation 6.7.2. The second one follows from the fact that multiplication by $p$ on $J_{\mathbb{F}_{p}}^{0}$ induces multiplication by $p$ on the tangent space at 0 , which is the zero map. Hence, the tangent space at 0 of $J_{\mathbb{F}_{p}}^{0}[p]$ is equal to the one of $J_{\mathbb{F}_{p}}^{0}$.

To establish an explicit link between parabolic cohomology and modular forms, we identify the parabolic cohomology group for $\Gamma_{1}(N)$ with $\mathbb{F}_{p}$-coefficients as the $p$-torsion of the Jacobian of the corresponding modular curve. Here we may view the Jacobian as a complex abelian variety. As in the other cases, the Hecke correspondences on the modular curves give rise to Hecke operators on the Jacobian.

Proposition 5.3 Let $N \geq 3$ be an integer, and $p$ a prime. Then there are isomorphisms of $\mathbb{F}_{p}$-vector spaces

$$
H_{\mathrm{par}}^{1}\left(\Gamma_{1}(N p), \mathbb{F}_{p}\right) \cong J(\mathbb{C})[p]=J\left(\overline{\mathbb{Q}}_{p}\right)[p]
$$

respecting the Hecke operators.

Proof. The second equality follows from the fact that torsion points are algebraic. We start with the exact Kummer sequence of analytic sheaves on $X_{1}(N p)$

$$
0 \rightarrow \mu_{p} \rightarrow \mathbb{G}_{m} \stackrel{p}{\rightarrow} \mathbb{G}_{m} \rightarrow 0 .
$$

Its long exact sequence in analytic cohomology yields

$$
0 \rightarrow H^{1}\left(X_{1}(N p), \mu_{p}\right) \rightarrow H^{1}\left(X_{1}(N p), \mathbb{G}_{m}\right) \stackrel{p}{\rightarrow} H^{1}\left(X_{1}(N p), \mathbb{G}_{m}\right) .
$$

Using $H^{1}\left(X_{1}(N p), \mathbb{G}_{m}\right) \cong \operatorname{Pic}_{X_{1}(N p)}(\mathbb{C})$ one obtains that $H^{1}\left(X_{1}(N p), \mu_{p}\right)$ is isomorphic to $J(\mathbb{C})[p]$. As $\mathbb{C}$ contains the $p$-th roots of unity, we may replace the sheaf $\mu_{p}$ by the constant sheaf $\mathbb{F}_{p}$. Moreover, the group $H^{1}\left(X_{1}(N p), \mathbb{F}_{p}\right)$ coincides with $H_{\mathrm{par}}^{1}\left(\Gamma_{1}(N p), \mathbb{F}_{p}\right)$. This follows for example from the Leray spectral sequence for the open immersion $Y_{1}(N p) \hookrightarrow X_{1}(N p)$ (see e.g. [23]). Since the Hecke operators come from correspondences on modular curves, the isomorphisms are compatible for the Hecke action.

\section{Hecke algebras}

In this section we compare the Hecke algebra of cuspidal modular forms to that of parabolic group cohomology and establish isomorphisms in certain cases. The principal result is Theorem 6.7 from which the application to ordinary cusp forms (Corollary 6.9) mentioned in the introduction follows. 
Whenever for a ring $R$ we have an $R$-module $M$, on which Hecke operators $T_{n}$ act for all $n$, we let

$$
\mathbb{T}_{R}(M):=R\left[T_{n} \mid n \in \mathbb{N}\right] \subseteq \operatorname{End}_{R}(M),
$$

i.e. the $R$-subalgebra of the endomorphism algebra generated by the Hecke operators.

We claim that in the situation of Hecke operators on modular forms or group cohomology for $\Gamma_{1}(N)$ of weight $k$ the diamond operators are in $\mathbb{T}_{R}(M)$. For $a$ coprime to $N$, let $l_{1}, l_{2}$ be distinct primes which are congruent to $a$ modulo $N$. Then $l_{i}^{k-1}\langle a\rangle=T_{l_{i}}^{2}-T_{l_{i}^{2}}$, so that with $1=r l_{1}^{k-1}+s l_{2}^{k-1}$ the claim follows.

\section{The Hecke algebra of modular forms and Eichler-Shimura}

We start by stating the Eichler-Shimura theorem (see [5], Theorem 12.2.2 and Proposition 12.4.10).

Theorem 6.1 (Eichler-Shimura) For $k \geq 2$ and $\Gamma \leq \mathrm{SL}_{2}(\mathbb{Z})$ a congruence subgroup there is an isomorphism of $\mathbb{T}_{\mathbb{Z}}\left(S_{k}(\Gamma, \mathbb{C})\right)$-modules, the Eichler-Shimura isomorphism,

$$
H_{\mathrm{par}}^{1}\left(\Gamma, V_{k-2}(\mathbb{C})\right) \cong S_{k}(\Gamma, \mathbb{C}) \oplus \overline{S_{k}(\Gamma, \mathbb{C})}
$$

Corollary 6.2 For $k \geq 2$ and $N \geq 3$ we have natural ring isomorphisms

$$
\mathbb{T}_{\mathbb{Z}}\left(S_{k}\left(\Gamma_{1}(N), \mathbb{C}\right)\right) \cong \mathbb{T}_{\mathbb{Z}}\left(H_{\text {par }}^{1}\left(\Gamma_{1}(N), V_{k-2}(\mathbb{Z})\right) / \text { torsion }\right) .
$$

Proof. The free $\mathbb{Z}$-module $H_{\mathrm{par}}^{1}\left(\Gamma_{1}(N), V_{k-2}(\mathbb{Z})\right) /$ torsion is a $\mathbb{Z}$-structure in the $\mathbb{C}$-vector space $H_{\text {par }}^{1}\left(\Gamma_{1}(N), V_{k-2}(\mathbb{C})\right)$. Any $\mathbb{Z}$-structure gives an isomorphic Hecke algebra. Finally, Theorem 6.1 implies that the Hecke algebra of $H_{\text {par }}^{1}\left(\Gamma_{1}(N), V_{k-2}(\mathbb{C})\right)$ is isomorphic to the Hecke algebra of $S_{k}\left(\Gamma_{1}(N), \mathbb{C}\right)$.

The formula in this corollary is the reason why many people prefer to factor out the torsion of modular symbols.

Proposition 6.3 Let $N \geq 5, k \geq 2$ integers and $p \nmid N$ a prime. Then we have

$$
\mathbb{T}_{\mathbb{Z}}\left(S_{k}\left(\Gamma_{1}(N), \mathbb{C}\right)\right) \otimes_{\mathbb{Z}} \mathbb{F}_{p} \cong \mathbb{T}_{\mathbb{F}_{p}}\left(S_{k}\left(\Gamma_{1}(N), \mathbb{F}_{p}\right)\right) .
$$

Proof. By [5], Theorem 12.3.2, there is no difference between Katz cusp forms over $\mathbb{F}_{p}$ and those that are reductions of classical cusp forms whose $q$ expansion is in $\mathbb{Z}[1 / N]$, i.e.

$$
S_{k}\left(\Gamma_{1}(N), \mathbb{Z}[1 / N]\right) \otimes_{\mathbb{Z}[1 / N]} \mathbb{F}_{p} \cong S_{k}\left(\Gamma_{1}(N), \mathbb{F}_{p}\right) .
$$

Hence, the $q$-expansion principle gives the two perfect pairings

$\mathbb{T}_{\mathbb{Z}}\left(S_{k}\left(\Gamma_{1}(N), \mathbb{C}\right)\right) \otimes_{\mathbb{Z}} \mathbb{Z}[1 / N] \times S_{k}\left(\Gamma_{1}(N), \mathbb{Z}[1 / N]\right) \rightarrow \mathbb{Z}[1 / N], \quad(T, f) \mapsto a_{1}(T f)$ 
and

$$
\mathbb{T}_{\mathbb{F}_{p}}\left(S_{k}\left(\Gamma_{1}(N), \mathbb{F}_{p}\right)\right) \times S_{k}\left(\Gamma_{1}(N), \mathbb{F}_{p}\right) \rightarrow \mathbb{F}_{p}, \quad(T, f) \mapsto a_{1}(T f) .
$$

Tensoring the first one with $\mathbb{F}_{p}$ allows us to compare it to the second one, from which the proposition follows.

Corollary 6.4 Let $p$ be a prime and $N \geq 5,2 \leq k \leq p+2$ integers s.t. $p \nmid N$. Then sending the operator $T_{l}$ to $T_{l}$ for all primes $l$ defines a surjective $\mathbb{F}_{p}$-algebra homomorphism

$$
\mathbb{T}_{\mathbb{F}_{p}}\left(S_{k}\left(\Gamma_{1}(N), \mathbb{F}_{p}\right)\right) \rightarrow \mathbb{T}_{\mathbb{F}_{p}}\left(H_{\mathrm{par}}^{1}\left(\Gamma_{1}(N), V_{k-2}\left(\mathbb{F}_{p}\right)\right)\right) .
$$

Proof. From Corollary 6.2 we obtain because of $p$-torsion-freeness (Proposition 2.6) an isomorphism of $\mathbb{F}_{p}$-algebras

$$
\mathbb{T}_{\mathbb{Z}}\left(S_{k}\left(\Gamma_{1}(N), \mathbb{C}\right)\right) \otimes \mathbb{F}_{p} \cong \mathbb{T}_{\mathbb{Z}}\left(H_{\mathrm{par}}^{1}\left(\Gamma_{1}(N), V_{k-2}(\mathbb{Z})\right)\right) \otimes_{\mathbb{Z}} \mathbb{F}_{p}
$$

By Proposition 6.3 the term on the left hand side is equal to $\mathbb{T}_{\mathbb{F}_{p}}\left(S_{k}\left(\Gamma_{1}(N), \mathbb{F}_{p}\right)\right)$ so that it suffices to have a surjection

$$
\mathbb{T}_{\mathbb{Z}}\left(H_{\mathrm{par}}^{1}\left(\Gamma_{1}(N), V_{k-2}(\mathbb{Z})\right)\right) \otimes \mathbb{F}_{p} \rightarrow \mathbb{T}_{\mathbb{F}_{p}}\left(H_{\mathrm{par}}^{1}\left(\Gamma_{1}(N), V_{k-2}\left(\mathbb{F}_{p}\right)\right)\right),
$$

which follows from Proposition 2.6. Indeed, the isomorphism

$$
H_{\text {par }}^{1}\left(\Gamma_{1}(N), V_{k-2}(\mathbb{Z})\right) \otimes \mathbb{F}_{p} \cong H_{\text {par }}^{1}\left(\Gamma_{1}(N), V_{k-2}\left(\mathbb{F}_{p}\right)\right)
$$

is compatible with Hecke operators, and allows one to define a homomorphism from the Hecke algebra on the left hand term to the one on the right hand term, which is automatically surjective by the definition of the Hecke algebra.

Proposition 6.5 Let $N \geq 1, k \geq 2$ be integers and $K$ a field. If the characteristic of $K$ is $p>0$, then we assume $p \nmid N$. Furthermore, let $\epsilon: \Gamma_{0}(N) \stackrel{\text { proj }}{\rightarrow}$ $\Gamma_{0}(N) / \Gamma_{1}(N) \rightarrow K^{*}$ be a character such that $\epsilon(-1)=(-1)^{k}$. Denote by $\mathbb{T}$ the $K$-Hecke algebra of $S_{k}\left(\Gamma_{1}(N), K\right)$ and by $\mathbb{T}_{\epsilon}$ the $K$-Hecke algebra of $S_{k}\left(\Gamma_{1}(N), \epsilon, K\right)$. Furthermore, let

$$
I=\left(\langle\delta\rangle-\epsilon(\delta) \mid \delta \in \Gamma_{0}(N) / \Gamma_{1}(N)\right) \triangleleft \mathbb{T} .
$$

Then $\mathbb{T} / I$ and $\mathbb{T}_{\epsilon}$ are isomorphic $K$-algebras.

Proof. As we work with Katz modular cusp forms (for that we need the condition $p \nmid N)$, we dispose of the $q$-expansion principle. Hence we have isomorphisms respecting the Hecke action $\left(\mathbb{T}_{\epsilon}\right)^{\vee} \cong S_{k}\left(\Gamma_{1}(N), \epsilon, K\right) \cong \mathbb{T}^{\vee}[I] \cong(\mathbb{T} / I)^{\vee}$, whence the proposition follows. 


\section{Comparing Hecke algebras over $\mathbb{F}_{p}$}

Proposition 6.6 Let $N \geq 5$ be an integer, $p \nmid N$ a prime and $0 \leq d \leq p-1$ an integer. There exists a surjection $\mathbb{T}_{\mathbb{F}_{p}}\left(H_{\text {par }}^{1}\left(\Gamma_{1}(N p), \mathbb{F}_{p}\right)(d)\right) \rightarrow \mathbb{T}_{\mathbb{F}_{p}}(\bar{L}(d))$ such that the diagram of $\mathbb{F}_{p}$-algebras

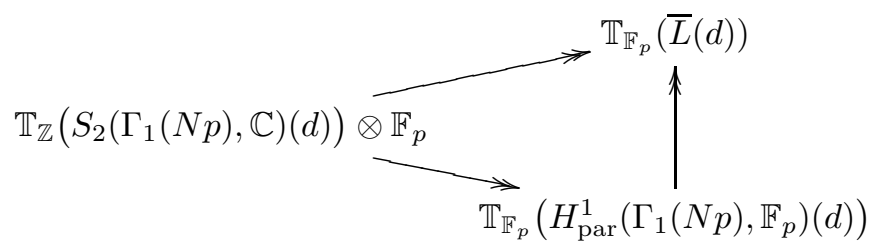

commutes. All maps are uniquely determined by sending the Hecke operator $T_{l}$ to $T_{l}$ for all primes $l$.

Proof. Let us first remark how the diagonal arrows are made. The lower one comes from the isomorphism (see Proposition 2.6)

$$
H_{\mathrm{par}}^{1}\left(\Gamma_{1}(N p), \mathbb{Z}\right) \otimes \mathbb{F}_{p} \cong H_{\mathrm{par}}^{1}\left(\Gamma_{1}(N p), \mathbb{F}_{p}\right) .
$$

The upper one is due to the fact that $L$ is a lattice in $S_{2}\left(\Gamma_{1}(N p), \mathbb{Q}_{p}\left(\zeta_{p}\right)\right.$ ) (see [11], p. 472) and using arguments as in Corollary 6.2 As the order of $\mathbb{F}_{p}^{*}$ is invertible in $\mathbb{F}_{p}$, we can everywhere pass to the eigencomponents of the action of the $p$-part of the diamond operator $\langle\cdot\rangle_{p}$.

We obtain the vertical arrow by showing that the kernel of the lower diagonal map is contained in the kernel of the upper diagonal map. In other words, we will show that if a Hecke operator $T$ in $\mathbb{T}_{\mathbb{Z}}\left(S_{2}\left(\Gamma_{1}(N p), \mathbb{C}\right)(d)\right) \otimes \mathbb{F}_{p}$ acts as zero on $H_{\mathrm{par}}^{1}\left(\Gamma_{1}(N p), \mathbb{F}_{p}\right)(d)$, then it acts as zero on $\bar{L}(d)$.

So assume that $T$ acts as zero on $H_{\text {par }}^{1}\left(\Gamma_{1}(N p), \mathbb{F}_{p}\right)(d)$. By Proposition 5.3 it acts as zero on $J_{\overline{\mathbb{Q}}_{p}}\left(\overline{\mathbb{Q}}_{p}\right)[p](d)$, hence on $J_{\overline{\mathbb{Q}}_{p}}[p](d)$, as $J_{\mathbb{Q}_{p}}[p]$ is reduced. But then it also acts as zero on $J_{\mathbb{Z}_{p}\left[\zeta_{p}\right]}[p](d)$, as it acts as zero on the generic fibre using that $J[p]$ is flat over $\mathbb{Z}_{p}\left[\zeta_{p}\right]$ ([2], Lemma 7.3.2, as $J$ is semi-abelian). But consequently, it also acts as zero on the special fibre $J_{\mathbb{F}_{p}}[p](d)$, whence also on the cotangent space $\operatorname{Cot}_{0}\left(J_{\mathbb{F}_{p}}^{0}[p]\right)(d)$. Now Lemma[5.2 finishes the proof.

Theorem 6.7 Let $p$ be a prime and $2<k \leq p+1, N \geq 5$ integers such that $p \nmid N$. We write for short $\mathbb{T}^{\text {par }, N, k}:=\mathbb{T}_{\mathbb{F}_{p}}\left(H_{\text {par }}^{1}\left(\Gamma_{1}(N), V_{k-2}\left(\mathbb{F}_{p}\right)\right)\right), \mathbb{T}^{\bmod , N, k}:=$ $\mathbb{T}_{\mathbb{F}_{p}}\left(S_{k}\left(\Gamma_{1}(N), \mathbb{F}_{p}\right)\right)$ and similarly for the twisted ones. Then there is the commutative diagram of $\mathbb{F}_{p}$-algebras

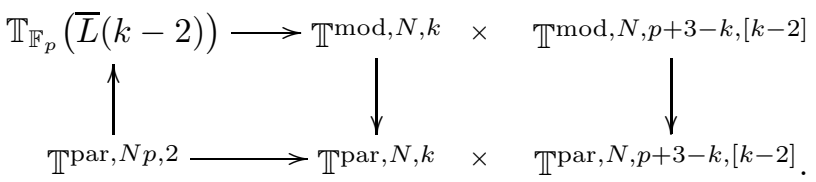

The vertical arrows are obtained from Proposition 6.6 resp. Corollary 6.4 and the horizontal ones from Proposition 5.1] and Proposition [4.2. The vertical arrows are surjective. If $2<k \leq p$, then the upper horizontal arrow is injective. 
Proof. The commutativity is clear, as $T_{l}$ is sent to $T_{l} \times T_{l}$ along the horizontal arrows, and $T_{l}$ is sent to $T_{l}$ along the vertical arrows for all primes $l$. The surjectivity of the vertical arrows has been proved at the places cited above. The injectivity of the upper homomorphism is the fact that $\bar{L}(k-2)$ is the direct sum of $S_{k}\left(\Gamma_{1}(N), \mathbb{F}_{p}\right)$ and $S_{p+3-k}\left(\Gamma_{1}(N), \mathbb{F}_{p}\right)[k-2]$, if $2<k \leq p$.

Corollary 6.8 Let $2<k \leq p+1, N \geq 5$ such that $p \nmid N$. Let $\mathfrak{P}$ be a maximal ideal of the Hecke algebra $\mathbb{T}_{\mathbb{Z}}\left(S_{2}\left(\Gamma_{1}(N p), \mathbb{C}\right)(d)\right) \otimes \mathbb{F}_{p}$ which is not in the support of $S_{p+3-k}\left(\Gamma_{1}(N), \mathbb{F}_{p}\right)$. Then we have an isomorphism

$$
\mathbb{T}_{\mathbb{F}_{p}}\left(S_{k}\left(\Gamma_{1}(N), \mathbb{F}_{p}\right)_{\mathfrak{P}}\right) \cong \mathbb{T}_{\mathbb{F}_{p}}\left(H_{\mathrm{par}}^{1}\left(\Gamma_{1}(N), V_{k-2}\left(\mathbb{F}_{p}\right)\right)_{\mathfrak{P}}\right) .
$$

Proof. The assumption means that $\left(S_{p+3-k}\left(\Gamma_{1}(N), \mathbb{F}_{p}\right)[k-2]\right)_{\mathfrak{P}}=0$. By Corollary 6.4 the ideal $\mathfrak{P}$ is not in the support of $H_{\mathrm{par}}^{1}\left(\Gamma_{1}(N), V_{p+1-k}\left(\mathbb{F}_{p}\right)\right)[k-2]$ either, whence $\left(H_{\text {par }}^{1}\left(\Gamma_{1}(N), V_{p+1-k}\left(\mathbb{F}_{p}\right)\right)[k-2]\right)_{\mathfrak{P}}=0$. Hence, the sequences of Propositions 4.2 and 5.1 localised at $\mathfrak{P}$ give isomorphisms $\mathbb{T}_{\mathbb{F}_{p}}(\bar{L}(k-2))_{\mathfrak{P}} \cong$ $\mathbb{T}_{\mathfrak{P}}^{\bmod , N, k}$ and $\mathbb{T}_{\mathfrak{P}}^{\text {par, }, N p, 2} \cong \mathbb{T}_{\mathfrak{P}}^{\text {par }, N, k}$. Hence, also the vertical maps in the localisation at $\mathfrak{P}$ of the diagram of Theorem 6.7 are isomorphisms.

Corollary 6.9 Let $2<k \leq p+1, N \geq 5$ such that $p \nmid N$. Let $\mathfrak{P}$ be a maximal ideal of $\mathbb{T}_{\mathbb{F}_{p}}\left(S_{k}\left(\Gamma_{1}(N), \mathbb{F}_{p}\right)\right)$ corresponding to a normalised eigenform $f \in S_{k}\left(\Gamma_{1}(N), \overline{\mathbb{F}_{p}}\right)$ which is ordinary, i.e. $a_{p}(f) \neq 0$. Then we have an isomorphism

$$
\mathbb{T}_{\mathbb{F}_{p}}\left(S_{k}\left(\Gamma_{1}(N), \mathbb{F}_{p}\right)_{\mathfrak{P}}\right) \cong \mathbb{T}_{\mathbb{F}_{p}}\left(H_{\text {par }}^{1}\left(\Gamma_{1}(N), V_{k-2}\left(\mathbb{F}_{p}\right)\right)_{\mathfrak{P}}\right) .
$$

Proof. As the operator $T_{p}$ always acts as zero on $S_{p+3-k}\left(\Gamma_{1}(N), \mathbb{F}_{p}\right)[k-2]$ the maximal ideal $\mathfrak{P}$ cannot be in the support of $S_{p+3-k}\left(\Gamma_{1}(N), \mathbb{F}_{p}\right)[k-2]$, whence we are in the situation of Corollary 6.8

\section{Application to $\Gamma_{0}(N)$ and characters}

The techniques from the preceding sections do not apply to the group $\Gamma_{0}(N)$. In this section we show that one can, nevertheless, derive similar results for that group together with a character of the quotient group $\Gamma_{0}(N) / \Gamma_{1}(N) \cong(\mathbb{Z} / N \mathbb{Z})^{*}$.

Let us for the sequel of this section make the following assumption.

Assumption 7.1 Let $p \geq 5$ be a prime and $K$ a finite field of characteristic $p$ or $K=\overline{\mathbb{F}_{p}}$. Suppose, moreover, that we are given integers $N \geq 5$ and $k$ with $3 \leq k \leq p+1$ and $p \nmid N$. Let $\Delta=\Gamma_{0}(N) / \Gamma_{1}(N)$. It acts on $H^{1}\left(\Gamma_{1}(N), \cdot\right)$, the parabolic subspace and on $S_{k}\left(\Gamma_{1}(N), K\right)$ through the diamond operators. Furthermore, let $\epsilon$ be a character of the form $\epsilon: \Gamma_{0}(N) \stackrel{\text { proj }}{\rightarrow} \Gamma_{0}(N) / \Gamma_{1}(N) \rightarrow K^{*}$. Denote by $K^{\epsilon}$ the $K\left[\Gamma_{0}(N)\right]$-module which is a copy of $K$ with action through $\epsilon^{-1}$. We write $V_{k-2}^{\epsilon}$ for the $K\left[\Gamma_{0}(N)\right]$-module $V_{k-2}(K) \otimes_{K} K^{\epsilon}$. Finally, let $G$ be the group with $\Gamma_{1}(N) \leq G \leq \Gamma_{0}(N)$ such that $G / \Gamma_{1}(N)=\Delta_{p}$, the $p$-Sylow subgroup of $\Delta$. Note that $G$ acts freely on $\mathbb{H}$. 
Lemma 7.2 Let us assume [7.1. Then $H^{1}\left(\Gamma_{1}(N), V_{k-2}(K)\right)$ and its parabolic subspace are coinduced $K\left[\Delta_{p}\right]$-modules.

Proof. We write $V=V_{k-2}(K)$ and $\Gamma:=\Gamma_{1}(N)$. The Hochschild-Serre spectral sequence (in the notation from [17, Appendix B, we use the exact sequence $E_{1}^{2} \rightarrow E_{2}^{1,1} \rightarrow E_{2}^{3,0}$ ) gives the exact sequence

$$
\begin{aligned}
\operatorname{ker}\left(H^{2}(G, V) \rightarrow H^{0}\left(\Delta_{p}, H^{2}(\Gamma, V)\right)\right) & \\
& \rightarrow H^{1}\left(\Delta_{p}, H^{1}(\Gamma, V)\right) \rightarrow H^{3}\left(\Delta_{p}, H^{0}(\Gamma, V)\right),
\end{aligned}
$$

whence $H^{1}\left(\Delta_{p}, H^{1}(\Gamma, V)\right)$ is zero by Corollary 2.1 and Proposition 2.5 From Proposition 2.2 it follows taking $\Delta_{p}$-cohomology that the sequence

$$
\begin{aligned}
0 & \rightarrow H_{\mathrm{par}}^{1}(\Gamma, V)^{\Delta_{p}} \rightarrow H^{1}(\Gamma, V)^{\Delta_{p}} \stackrel{\text { res }}{\longrightarrow}\left(\prod_{g \in \Gamma \backslash \mathrm{PSL}_{2}(\mathbb{Z}) /\langle T\rangle} H^{1}\left(\Gamma \cap\left\langle g T g^{-1}\right\rangle, V\right)\right)^{\Delta_{p}} \\
& \rightarrow H^{1}\left(\Delta_{p}, H_{\mathrm{par}}^{1}(\Gamma, V)\right) \rightarrow 0
\end{aligned}
$$

is exact. It can be checked (using that $-1 \notin G$ and that $p$ does not divide the ramification indices of the cusps) that the first three terms are

$$
0 \rightarrow H_{\mathrm{par}}^{1}(G, V) \rightarrow H^{1}(G, V) \stackrel{\text { res }}{\longrightarrow} \prod_{g \in G \backslash \mathrm{PSL}_{2}(\mathbb{Z}) /\langle T\rangle} H^{1}\left(G \cap\left\langle g T g^{-1}\right\rangle, V\right) \rightarrow 0,
$$

where the zero on the right is again a consequence of Proposition 2.2. Hence, $H^{1}\left(\Delta_{p}, H_{\mathrm{par}}^{1}(\Gamma, V)\right)$ is zero. Finally, 19, Proposition 1.7.3(ii), implies that $H^{1}(\Gamma, V)$ and its parabolic subspace are coinduced $\Delta_{p}$-modules.

Lemma 7.3 Let us assume 7.1. Then $S_{k}\left(\Gamma_{1}(N), K\right)$ is a free $K\left[\Delta_{p}\right]$-module.

Proof. For the notation in this proof we follow [6], pp. 209-210 and the proof of Lemma 1.9. We let $\Gamma:=\Gamma_{1}(N)$.

The projection $\pi: X_{\Gamma} \rightarrow X_{G}$ is a Galois cover with group $\Delta_{p}$ of projective $K$ schemes. Indeed, for the open part this is [4, VI.2.7. Moreover, the ramification index of the cusps divides $N$, whence the cusps are unramified in a $p$-extension.

Using Serre duality and the Kodaira-Spencer isomorphism (see [4], VI.4.5.2) we obtain

$$
\begin{aligned}
H^{1}\left(X_{G}, \omega^{\otimes k}(- \text { cusps })\right) & \stackrel{\text { S-D }}{\cong} H^{0}\left(X_{G}, \Omega^{1} \otimes\left(\omega^{\otimes k}(- \text { cusps })\right)^{\vee}\right)^{\vee} \\
& \stackrel{\text { K-S }}{\cong} H^{0}\left(X_{G}, \omega^{\otimes 2-k}\right)^{\vee}
\end{aligned}
$$

which is zero, since the degree of $\omega^{\otimes 2-k}$ is negative (as $k \geq 3$ ). The map $\pi$ is étale and we have $H^{0}\left(X_{\Gamma}, \pi^{*} \omega^{\otimes k}(-\right.$ cusps $\left.)\right) \cong S_{k}(\Gamma, K)$. We conclude from [18], Theorem 2, that this is a free $K\left[\Delta_{p}\right]$-module.

Theorem 7.4 Let us assume 7.1 and that $H_{\mathrm{par}}^{1}\left(\Gamma_{1}(N), V_{k-2}(K)\right)$ is a faithful $\mathbb{T}_{K}\left(S_{k}\left(\Gamma_{1}(N), K\right)\right)$-module. Then $H_{\mathrm{par}}^{1}\left(\Gamma_{0}(N), V_{k-2}^{\epsilon}\right)$ is a faithful module for $\mathbb{T}_{K}\left(S_{k}\left(\Gamma_{1}(N), \epsilon, K\right)\right)$. 
Proof. Let $\Gamma:=\Gamma_{1}(N)$. We claim that $N_{\Delta}:=\sum_{\delta \in \Delta} \delta \in K[\Delta]$ induces isomorphisms

$$
\left(S_{k}(\Gamma, K) \otimes_{K} K^{\epsilon}\right)_{\Delta} \rightarrow\left(S_{k}(\Gamma, K) \otimes_{K} K^{\epsilon}\right)^{\Delta}
$$

and

$$
H_{\mathrm{par}}^{1}\left(\Gamma, V_{k-2}^{\epsilon}\right)_{\Delta} \rightarrow H_{\mathrm{par}}^{1}\left(\Gamma, V_{k-2}^{\epsilon}\right)^{\Delta} .
$$

We note that $H^{1}\left(\Gamma, V_{k-2}(K)\right) \otimes_{K} K^{\epsilon}=H^{1}\left(\Gamma, V_{k-2}^{\epsilon}\right)$, since the character $\epsilon$ restricted to $\Gamma$ is trivial. Using Lemmas 7.2 and 7.3 an elementary calculation gives the claim.

Dualising Equation (7.8) gives an isomorphism

$$
\left(\mathbb{T}\left(S_{k}(\Gamma, K)\right) \otimes K^{\epsilon}\right)_{\Delta} \stackrel{N_{\Delta}}{\longrightarrow}\left(\mathbb{T}\left(S_{k}(\Gamma, K)\right) \otimes K^{\epsilon}\right)^{\Delta},
$$

which in particular yields the implication

$$
T\left(\sum_{\delta \in \Delta} \epsilon(\delta)^{-1}\langle\delta\rangle\right)=0 \quad \Rightarrow \quad T \in I,
$$

where $I$ is the ideal defined in Proposition 6.5 In view of that proposition, we only need to show that if $T$ acts as zero on $H^{1}\left(G, V_{k-2}^{\epsilon}\right)$, then $T$ is in $I$.

The Hochschild-Serre spectral sequence yields $H^{1}\left(\Gamma, V_{k-2}^{\epsilon}\right)^{\Delta} \cong H^{1}\left(G, V_{k-2}^{\epsilon}\right)$, since $\left(V_{k-2}^{\epsilon}\right)^{\Gamma}$ is zero by Proposition 2.5. Let now $T$ be a Hecke operator. Then we have

$$
\begin{aligned}
T \cdot H^{1}\left(G, V_{k-2}^{\epsilon}\right) & =T \cdot H^{1}\left(\Gamma, V_{k-2}^{\epsilon}\right)^{\Delta} \\
& =T N_{\Delta} \cdot H^{1}\left(\Gamma, V_{k-2}^{\epsilon}\right)_{\Delta}=T\left(\sum_{\delta \in \Delta} \epsilon(\delta)^{-1}\langle\delta\rangle\right) \cdot H^{1}\left(\Gamma, V_{k-2}(K)\right)
\end{aligned}
$$

Suppose that this is zero. Hence, by the assumed faithfulness we have that $T\left(\sum_{\delta \in \Delta} \epsilon(\delta)^{-1}\langle\delta\rangle\right)=0$, which by Equation (7.10) implies $T \in I$, as required.

Remark 7.5 If $k=2$, then the statements of Theorem 7.4 also hold "outside the Eisenstein part". The Eisenstein part is the subspace on which the Hecke algebra acts via a system of eigenvalues that does not belong to an irreducible Galois representation. For, a suitable analogue of Lemma 7.3 holds, since $H^{1}\left(X_{G}, \omega^{\otimes k}(-\right.$ cusps $\left.)\right) \cong H^{0}\left(X_{G}, \mathcal{O}\right)^{\vee}$ cannot give rise to a non-Eisenstein system of eigenvalues. Moreover, under the extra assumption also Lemma 7.2 remains valid, as an easy calculation shows.

\section{Application to weight one modular forms}

Edixhoven explains in [8], Section 4, how weight one cuspidal Katz modular forms over finite fields of characteristic $p$ can be computed from the knowledge of the Hecke algebra of weight $p$ cusp forms over the same field. In this section 
we shall first recall this and then derive the conclusion mentioned in the introduction that weight one modular cusp forms over $\overline{\mathbb{F}_{p}}$ can be computed by the Hecke operators on weight $p$ parabolic group cohomology over $\mathbb{F}_{p}$.

Let $\mathbb{F}$ be a finite field of prime characteristic $p$ or $\overline{\mathbb{F}_{p}}$ and fix a level $N \geq 1$ with $p \nmid N$ and a character $\epsilon:(\mathbb{Z} / N \mathbb{Z})^{*} \rightarrow \mathbb{F}^{*}$ with $\epsilon(-1)=(-1)^{k}$. We have two injections of $\mathbb{F}$-vector spaces

$$
F, A: S_{1}\left(\Gamma_{1}(N), \epsilon, \mathbb{F}\right) \rightarrow S_{p}\left(\Gamma_{1}(N), \epsilon, \mathbb{F}\right),
$$

given on $q$-expansions by $a_{n}(A g)=a_{n}(g)$ and $a_{n}(F g)=a_{n / p}(g)$ (with $a_{n}(F g)=$ 0 if $p \nmid n)$, which are compatible with all Hecke operators $T_{l}$ for primes $l \neq p$. The former comes from the Frobenius and the latter is multiplication by the Hasse invariant. One has $T_{p}^{(p)} F=A$ and $A T_{p}^{(1)}=T_{p}^{(p)} A+\epsilon(p) F$, where we have indicated the weight as a superscript (see e.g. [8], Equation (4.1.2)).

Let $\mathbb{T}^{(k)}$ be the Hecke algebra over $\mathbb{F}$ of weight $k$ for a fixed level $N$ and a fixed character $\epsilon$. We will also indicate the weight of Hecke operators by superscripts. We denote by $A^{(p)}$ the $\mathbb{F}_{p^{-}}$subalgebra of $\mathbb{T}^{(p)}$ generated by all Hecke operators $T_{n}^{(p)}$ for $p \nmid n$.

Proposition 8.1 (a) There is a homomorphism $\Theta$, called a derivation, which on q-expansions is given by $a_{n}(\Theta f)=n a_{n}(f)$ such that the sequence

$$
0 \rightarrow S_{1}\left(\Gamma_{1}(N), \epsilon, \mathbb{F}\right) \stackrel{F}{\rightarrow} S_{p}\left(\Gamma_{1}(N), \epsilon, \mathbb{F}\right) \stackrel{\Theta}{\rightarrow} S_{p+2}\left(\Gamma_{1}(N), \epsilon, \mathbb{F}\right)
$$

is exact.

(b) Suppose $f \in S_{1}\left(\Gamma_{1}(N), \epsilon, \mathbb{F}\right)$ such that $a_{n}(f)=0$ for all $n$ with $p \nmid n$. Then $f=0$. In particular $A S_{1}\left(\Gamma_{1}(N), \epsilon, \mathbb{F}\right) \cap F S_{1}\left(\Gamma_{1}(N), \epsilon, \mathbb{F}\right)=0$.

(c) The Hecke algebra $\mathbb{T}^{(1)}$ in weight one can be generated by all $T_{l}^{(1)}$, where $l$ runs through the primes different from $p$.

(d) The weight one Hecke algebra $\mathbb{T}^{(1)}$ is the algebra generated by the $A^{(p)}$-action on the module $\mathbb{T}^{(p)} / A^{(p)}$.

Proof. (a) The main theorem of [15] gives the exact sequence

$$
0 \rightarrow S_{1}\left(\Gamma_{1}(N), \epsilon, \mathbb{F}\right) \stackrel{F}{\longrightarrow} S_{p}\left(\Gamma_{1}(N), \epsilon, \mathbb{F}\right) \stackrel{A \Theta}{\longrightarrow} S_{2 p+1}\left(\Gamma_{1}(N), \epsilon, \mathbb{F}\right)
$$

by taking Galois invariants. However, as explained in [8], Section 4, the image $A \Theta S_{p}\left(\Gamma_{1}(N), \epsilon, \mathbb{F}\right)$ in weight $2 p+1$ can be divided by the Hasse invariant, whence the weight is as claimed.

(b) The condition implies by looking at $q$-expansions that $A \Theta f=0$, whence by Part (3) of Katz' theorem cited above $f$ comes from a lower weight than 1 , but below there is just the 0-form (see also [8], Proposition 4.4).

(c) It is enough to show that $T_{p}^{(1)}$ is linearly dependent on the span of all $T_{n}^{(1)}$ for $p \nmid n$. If it were not, then there would be a modular cusp form of weight 1 satisfying $a_{n}(f)=0$ for $p \nmid n$, but $a_{p}(f) \neq 0$, contradicting (b).

(d) Dualising the exact sequence in (a) yields that $\mathbb{T}^{(p)} / A^{(p)}$ and $\mathbb{T}^{(1)}$ are isomorphic as $A^{(p)}$-modules, which implies the claim. 
Proposition 8.2 Let $N \geq 1$ and $k \geq 2$ be integers such that $p \nmid N, \mathbb{F} \mid \mathbb{F}_{p}$ a finite extension and let $\epsilon:(\mathbb{Z} / N \mathbb{Z})^{*} \rightarrow \mathbb{F}^{*}$ be a character with $\epsilon(-1)=(-1)^{k}$. Set

$$
B=\frac{N}{12} \prod_{l \mid N, l \text { prime }}\left(1+\frac{1}{l}\right) .
$$

(a) Then the Hecke operators $T_{1}^{(k)}, T_{2}^{(k)}, \ldots, T_{k B}^{(k)}$ generate $\mathbb{T}^{(k)}$ as an $\mathbb{F}$-vector space.

(b) The $\mathbb{F}$-algebra $A^{(p)}$ can already be generated as an $\mathbb{F}$-vector space by the set

$$
\left\{T_{n}^{(p)} \mid p \nmid n, n \leq(p+2) B\right\} .
$$

Proof. (a) This follows from the proof of [8, Proposition 4.2.

(b) Assume that some $T_{m}^{(p)}$ for $m>(p+2) B$ and $p \nmid m$ is linearly independent of the operators in the set of the assertion. This means that there is a cusp form $f \in S_{p}\left(\Gamma_{1}(N), \epsilon, \mathbb{F}\right)$ satisfying $a_{n}(f)=0$ for all $n \leq(p+2) B$ with $p \nmid n$, but $a_{m}(f) \neq 0$. One gets $a_{n}(\Theta f)=0$ for all $n \leq(p+2) B$, but $a_{m}(\Theta f) \neq 0$. This contradicts (a).

Remark 8.3 If we work with $\Gamma_{1}(N)$ and no character, the number $B$ above has to be replaced by

$$
B^{\prime}=\frac{N^{2}}{24} \prod_{l \mid N, l \text { prime }}\left(1-\frac{1}{l^{2}}\right) .
$$

Part of the following proposition is $[8$, Proposition 6.2.

Proposition 8.4 Let $V \subset S_{p}\left(\Gamma_{1}(N), \epsilon, \overline{\mathbb{F}}\right)$ be the eigenspace of a system of eigenvalues for the operators $T_{l}^{(p)}$ for all primes $l \neq p$

If the system of eigenvalues does not come from a weight one form, then $V$ is at most of dimension one. Conversely, if there is a normalised weight one eigenform $g$ with that system of eigenvalues for $T_{l}^{(1)}$ for all primes $l \neq p$, then $V=\langle A g, F g\rangle$ and that space is 2-dimensional. On it $T_{p}^{(p)}$ acts with eigenvalues $u$ and $\epsilon(p) u^{-1}$ satisfying $u+\epsilon(p) u^{-1}=a_{p}(g)$. In particular, the eigenforms in weight $p$ which come from weight one are ordinary.

Proof. We choose a normalised eigenform $f$ for all operators. If $V$ is at least 2-dimensional, then we have $V=\mathbb{F} f \oplus\left\{h \mid a_{n}(h)=0 \forall p \nmid n\right\}$. As a form $h$ in the right summand is annihilated by $\Theta$, it is equal to $F g$ for some form $g$ of weight one by Proposition 8.1 (a). By Part (b) of that proposition we know that $\langle A g, F g\rangle$ is 2-dimensional. If $V$ were more than 2-dimensional, then there would be two different cusp forms in weight 1 , which are eigenforms for all $T_{l}^{(1)}$ with $l \neq p$. This, however, contradicts Part (c).

Assume now that $V$ is 2-dimensional. Any normalised eigenform $f \in V$ for all Hecke operators in weight $p$ has to be of the form $A g+\mu F g$ for some $\mu \in \overline{\mathbb{F}}$. 
The eigenvalue of $T_{p}^{(p)}$ on $f$ is the $p$-th coefficient, hence $u=a_{p}(g)+\mu$, as $a_{p}(F g)=a_{1}(g)=1$. Now we have

$$
\begin{aligned}
\left(a_{p}(g)+\mu\right)(A g+\mu F g) & =T_{p}^{(p)}(A g+\mu F g)=T_{p}^{(p)} A g+\mu A g \\
& =A T_{p}^{(1)} g-\epsilon(p) F g+\mu A g=\left(a_{p}(g)+\mu\right) A g-\epsilon(p) F g
\end{aligned}
$$

which implies $-\epsilon(p)=\left(a_{p}(g)+\mu\right) \mu=u^{2}-u a_{p}(g)$ by looking at the $p$-th coefficient. From this one obtains the claim on $u$.

Theorem 8.5 Let $N \geq 5$ an integer and $p$ be a prime not dividing $N$.

(a) The Hecke algebra of $S_{1}\left(\Gamma_{1}(N), \mathbb{F}_{p}\right)$ can be computed using the first $(p+2) B^{\prime}$ Hecke operators on $H_{\mathrm{par}}^{1}\left(\Gamma_{1}(N), V_{p-2}\left(\mathbb{F}_{p}\right)\right)$.

(b) Let $\mathbb{F}$ be a finite field of characteristic $p \geq 5$ and let $\epsilon:(\mathbb{Z} / N \mathbb{Z})^{*} \rightarrow \mathbb{F}^{*}$ be a character. The Hecke algebra of $S_{1}\left(\Gamma_{1}(N), \epsilon, \mathbb{F}\right)$ can be computed using the first $(p+2) B$ Hecke operators on $H_{\mathrm{par}}^{1}\left(\Gamma_{0}(N), V_{p-2}^{\epsilon}(\mathbb{F})\right)$.

The numbers $B$ resp. $B^{\prime}$ were defined in Proposition 8.2 and Remark 8.3

Proof. (a) Corollary 6.9 implies that the ordinary part of $H_{\text {par }}^{1}\left(\Gamma_{1}(N), \mathbb{F}_{p}\right)$ is a faithful module for the ordinary part of the Hecke algebra of weight $p$ Katz cusp forms over $\mathbb{F}_{p}$. So that part of the Hecke algebra can be computed using the Hecke operators $T_{1}, \ldots, T_{p B^{\prime}}$ on $H_{\text {par }}^{1}\left(\Gamma_{1}(N), \mathbb{F}_{p}\right)$ (see Proposition 8.2(a)). From Proposition 8.4 we know that the image of the weight one forms in weight $p$ under the Hasse invariant and Frobenius lies in the ordinary part. Proposition 8.2 (b) implies that $A^{(p)}$ can be computed by the Hecke operators indicated there. Now the Hecke algebra of weight one Katz cusp forms on $\Gamma_{1}(N)$ without a character can be computed as described in Proposition 8.11 (d).

(b) Under the extra assumption and using Theorem 7.4 the same arguments also work with a character and the bound $B$.

\section{References}

[1] A. Ash and G. Stevens. Modular forms in characteristic $l$ and special values of their L-functions, Duke Math. J. 53 (1986), no. 3, 849-868.

[2] S. Bosch, W. Lütkebohmert and M. Raynaud. Néron Models, Springer Verlag, Berlin, 1990.

[3] K. S. Brown. Cohomology of groups, Springer, New York, 1982.

[4] P. Deligne and M. Rapoport. Les schémas de modules de courbes elliptiques, in Modular functions of one variable, II (Proc. Internat. Summer School, Univ. Antwerp, Antwerp, 1972), 143-316. Lecture Notes in Math. 349, Springer, Berlin, 1973.

[5] F. Diamond and J. Im. Modular forms and modular curves, in Seminar on Fermat's Last Theorem (Toronto, ON, 1993-1994), 39-133, Amer. Math. Soc., Providence, RI, 1995. 
[6] S. J. Edixhoven. Serre's Conjecture, in Modular forms and Fermat's last theorem (Boston, MA, 1995), 209-242, Springer, New York, 1997.

[7] S. J. Edixhoven. The weight in Serre's conjectures on modular forms, Invent. math. 109 (1992), no. 3, 563-594.

[8] S. J. Edixhoven. Comparison of integral structures on spaces of modular forms of weight two, and computation of spaces of forms mod 2 of weight one. Journal of the Inst. of Math. Jussieu 5 (2006), no. 1, 1-34.

[9] M. Emerton, R. Pollack and T. Weston. Variation of Iwasawa Invariants in Hida Families. Invent. math. 163 (2006), no. 3, 523-580.

$[10]$ G. Faltings, B. W. Jordan. Crystalline Cohomology and $\mathrm{GL}_{2}(\mathbb{Q})$, Israel J. Math. 90 (1995), no. 1-3, 1-66.

[11] B. H. Gross. A tameness criterion for Galois representations associated to modular forms $(\bmod p)$, Duke Math. J. $61(1990)$, no. 2, 445-517.

[12] A. Herremans. A combinatorial interpretation of Serre's conjecture on modular Galois representations, Ann. Inst. Fourier (Grenoble) 53 (2003), no. 5, $1287-1321$.

[13] P. J. Hilton, U. Stammbach. A course in homological algebra. Second edition. Graduate Texts in Mathematics, 4. Springer-Verlag, New York, 1997.

[14] N. M. Katz. p-adic properties of modular schemes and modular forms. Modular functions of one variable, III (Proc. Internat. Summer School, Univ. Antwerp, Antwerp, 1972). Lecture Notes in Mathematics, Vol. 350, Springer, Berlin, 1973, 69-190.

[15] N. M. Katz. A result on modular forms in characteristic $p$. Modular functions of one variable, V (Proc. Second Internat. Conf., Univ. Bonn, Bonn, 1976), 53-61. Lecture Notes in Math., Vol. 601, Springer, Berlin, 1977.

[16] N. M. Katz and B. Mazur. Arithmetic moduli of elliptic curves, Ann. of Math. Stud. 108, Princeton Univ. Press, Princeton, NJ, 1985.

[17] J. S. Milne. Etale cohomology. Princeton Mathematical Series, 33. Princeton University Press, Princeton, N.J., 1980.

[18] S. Nakajima. On Galois module structure of the cohomology groups of an algebraic variety. Inv. math. 75 (1984), 1-8.

[19] J. Neukirch, A. Schmidt and K. Wingberg. Cohomology of Number Fields, Grundlehren der mathematischen Wissenschaften 323, Springer, Berlin, 2000.

[20] G. Shimura. Introduction to the Arithmetic Theory of Automorphic Forms. Princeton University Press, 1994.

[21] G. Wiese. Computing Hecke algebras of weight 1 in Magma. Appendix B of 8 .

[22] G. Wiese. Modular Forms of Weight One Over Finite Fields. Ph.D. thesis, Universiteit Leiden.

[23] G. Wiese. On modular symbols and the cohomology of Hecke triangle surfaces. Preprint. arXiv:math.NT/0511113. 
[24] A. J. Wiles. Modular Elliptic Curves and Fermat's Last Theorem, Ann. of Math. (2) 141 (1995), no. 3, 443-551.

Gabor Wiese

NWF I - Mathematik

Universität Regensburg

D-93040 Regensburg

Deutschland

E-mail: gabor@pratum.net

Web page: http://maths.pratum.net/ 